CUBO A Mathematical Journal Vol.13, № 03, (69-89). October 2011

\title{
Lightlike Geometry of Leaves in Indefinite Kenmotsu Manifolds
}

\author{
Fortuné Massamba \\ Department of Mathematics, \\ University of Botswana, \\ Private Bag 0022 Gaborone, Botswana. \\ email: massfort@yahoo.fr, massambaf@mopipi.ub.bw
}

\begin{abstract}
In this paper, we study some aspects of the geometry of leaves of integrable distributions of lightlike hypersurfaces in indefinite Kenmotsu manifolds, tangent to the structure vector field. Theorems on parallel vector field, Killing distribution, geodesibility of lightlike hypersurfaces are obtained. Some characterization Theorems of leaves of integrable distributions are given. We prove that there exists a distribution, subset of the screen distribution, in which, under the integrability condition, any totally contact umbilical leaf is an extrinsic sphere.
\end{abstract}

\section{RESUMEN}

En este trabajo se estudian algunos aspectos de la geometría de las hojas de distribuciones integrables de las hipersuperficies luminosas en variedades de Kenmotsu indefinidas, tangentes a la estructura de un campo vectorial. Se obtienen algunos teoremas sobre el campo de vectores paralelo, distribución de Killing y geodesbilidad de hipersuperficies liminosas. Se dan algunos teoremas de caracterización de las hojas de las distribuciones integrables. Se demuestra que existe una distribucin, subconjunto de 
la distribucin de la pantalla, en la que, bajo la condición de integrabilidad, cualquier contacto con la hoja umbilical es una esfera extrínseca.

Keywords: Lightlike hypersurface; Indefinite Kenmotsu manifold; Screen distribution.

Mathematics Subject Classification: 53C15, 53C25, 53C40, 53C50.

\section{Introduction}

Several authors have studied some properties of Kenmotsu manifolds. In [7, for instance, the authors partially classified the Kenmotsu manifolds and considered manifolds admitting the transformation which keeps the Riemannian curvature tensor and Ricci tensor invariant.

The present paper aims to investigate the geometry of lightlike hypersurfaces of indefinite Kenmotsu manifolds, tangent to the structure vector field, with specific attention to the geometry of leaves of its integrable distributions.

As is well known, the geometry of lightlike submanifolds [4 are different because of the fact that their normal vector bundle intersects with the tangent bundle. Thus, the study becomes more difficult and strikingly different from the study of non-degenerate submanifolds. This means that one cannot use, in the usual way, the classical submanifold theory to define any induced object on a lightlike submanifold. To deal with this anomaly, the lightlike submanifolds were introduced and presented in a book by Duggal and Bejancu [4. They introduced a non-degenerate screen distribution to construct a nonintersecting lightlike transversal vector bundle of the tangent bundle. Several authors have studied lightlike hypersurfaces of semi-Riemannian manifolds (see [5] and many more references therein).

Physically, lightlike hypersurfaces are interesting in general relativity since they produce models of different types of horizons. On the Latter, the relationship between Killing and geodesic notions is well specified.

In [4, the authors discussed the Cauchy Riemann (CR) lightlike submanifolds of indefinite Käehler manifolds in (4, chapter 6) and proved that, in a totally umbilical real lightlike hypersurface of an indefinite Käehler space form, the nonzero mean curvature vector satisfies partial differential equations which imply that the nonzero mean curvature vector is not parallel. The usual terminology says that such an umbilical lightlike submanifold is not an extrinsic sphere (see 3. for more details). As the notion of totally umbilical submanifolds of Kaehlerian manifolds corresponds to that of totally contact umbilical submanifolds of Sasakian manifolds [10], the author in [13. showed that, in a totally contact umbilical lightlike hypersurface of an indefinite Sasakian space form, the nonzero mean curvature vector also is not parallel. But in [15] it is proved that any totally contact umbilical leaf of a screen integrable distribution of a lightlike hypersurface in an indefinite Sasakian space form is an extrinsic sphere.

Considering the mentioned notions above and those given in [6], 11], 12] and [14] on lightlike 
hypersurfaces of indefinite Sasakian manifolds, similar research is needed for the geometry of leaves of distribution of lightlike hypersurfaces in indefinite Kenmotsu manifolds. It is important to note that Kenmotsu manifolds are different from Sasakian manifolds.

The paper is organized as follows. In Section 2, we recall some basic definitions for indefinite Kenmotsu manifolds and lightlike hypersurfaces of semi-Riemannian manifolds. In Section 3 , we give a decomposition of almost contact metric of lightlike hypersurfaces in indefinite Kenmotsu manifolds, tangent to the structure vector field, supported by an example, as well as Theorems on Lie derivatives and parallel vector field. In Section 4, we investigate the geometry of leaves of integrable distributions of lightlike hypersurfaces. We prove that, if a leaf $M^{\prime}$ of the integrable distribution $\mathrm{D}_{0} \perp\langle\xi\rangle$ is totally geodesic, then $\bar{\phi}\left(\mathrm{TM}^{\perp}\right) \oplus \bar{\phi}(\mathrm{N}(\mathrm{TM}))$ is a Killing distribution. Moreover, if the second fundamental form of $M^{\prime}$ is parallel with respect to the Levi-Civita connection $\nabla^{\prime}$, then $M^{\prime}$ is totally geodesic (Theorem 4.3). A characterization of a leaf of $\mathrm{D}_{0} \perp\langle\xi\rangle$ is given (Theorem 4.4). We show that any totally contact umbilical leaf of an integrable distribution $\widehat{\mathrm{D}}$ of a lightlike hypersurface is an extrinsic sphere (Theorem 4.5).

\section{Preliminaries}

Let $\bar{M}$ be a $(2 n+1)$-dimensional manifold endowed with an almost contact structure $(\bar{\phi}, \xi, \eta)$, i.e. $\bar{\phi}$ is a tensor field of type $(1,1), \xi$ is a vector field, and $\eta$ is a 1 -form satisfying

$$
\bar{\phi}^{2}=-\mathbb{I}+\eta \otimes \xi, \eta(\xi)=1, \eta \circ \bar{\phi}=0 \text { and } \bar{\phi} \xi=0 .
$$

Then $(\bar{\phi}, \xi, \eta, \bar{g})$ is called an indefinite almost contact metric structure on $\bar{M}$ if $(\bar{\phi}, \xi, \eta)$ is an almost contact structure on $\bar{M}$ and $\bar{g}$ is a semi-Riemannian metric on $\bar{M}$ such that, for any vector field $\bar{X}, \bar{Y}$ on $\bar{M}$ (see 2] for Riemannian case)

$$
\eta(\bar{X})=\bar{g}(\xi, \bar{X}), \quad \bar{g}(\bar{\phi} \bar{X}, \bar{\phi} \bar{Y})=\bar{g}(\bar{X}, \bar{Y})-\eta(\bar{X}) \eta(\bar{Y}) .
$$

If, moreover, $(\bar{\nabla} \bar{X} \bar{\phi}) \bar{Y}=\bar{g}(\bar{\phi} \bar{X}, \bar{Y}) \xi-\eta(\bar{Y}) \bar{\phi} \bar{X}$, where $\bar{\nabla}$ is the Levi-Civita connection for the semi-Riemannian metric $\bar{g}$, we call $\bar{M}$ an indefinite Kenmotsu manifold [9].

A plane section $\sigma$ in $T_{p} \bar{M}$ is called a $\bar{\phi}$-section if it is spanned by $\bar{X}$ and $\bar{\phi} \bar{X}$, where $\bar{X}$ is a unit tangent vector field orthogonal to $\xi$. The sectional curvature of a $\bar{\phi}$-section $\sigma$ is called a $\bar{\phi}$-sectional curvature. If a Kenmotsu manifold $\bar{M}$ has constant $\bar{\phi}$-sectional curvature c, then, by virtue of the Proposition 12 in [9], the curvature tensor $\bar{R}$ of $\bar{M}$ is given by

$$
\begin{aligned}
& \bar{R}(\bar{X}, \bar{Y}) \bar{Z}=\frac{c-3}{4}\{\bar{g}(\bar{Y}, \bar{Z}) \bar{X}-\bar{g}(\bar{X}, \bar{Z}) \bar{Y}\}+\frac{c+1}{4}\{\eta(\bar{X}) \eta(\bar{Z}) \bar{Y} \\
& -\eta(\bar{Y}) \eta(\bar{Z}) \bar{X}+\bar{g}(\bar{X}, \bar{Z}) \eta(\bar{Y}) \xi-\bar{g}(\bar{Y}, \bar{Z}) \eta(\bar{X}) \xi+\bar{g}(\bar{\phi} \bar{Y}, \bar{Z}) \bar{\phi} \bar{X} \\
& -\bar{g}(\bar{\phi} \bar{X}, \bar{Z}) \bar{\phi} \bar{Y}-2 \bar{g}(\bar{\phi} \bar{X}, \bar{Y}) \bar{\phi} \bar{Z}\}, \bar{X}, \bar{Y}, \bar{Z} \in \Gamma(T \bar{M}) .
\end{aligned}
$$

A Kenmotsu manifold $\bar{M}$ of constant $\bar{\phi}$-sectional curvature c will be called Kenmotsu space form and denoted $\bar{M}(\mathrm{c})$. 
Let $(\bar{M}, \bar{g})$ be a $(2 n+1)$-dimensional semi-Riemannian manifold with index $s, 0<s<2 n+1$ and let $(M, g)$ be a hypersurface of $\bar{M}$, with $g=\bar{g}_{\mid M}$. $M$ is a lightlike hypersurface of $\bar{M}$ if $g$ is of constant rank $2 \mathrm{n}-1$ and the normal bundle $\mathrm{TM}^{\perp}$, defined as

$$
\mathrm{TM}^{\perp}=\bigcup_{p \in M}\left\{\mathrm{Y}_{p} \in \mathrm{T}_{\mathrm{p}} \bar{M}: \bar{g}_{\mathrm{p}}\left(\mathrm{X}_{\mathrm{p}}, \mathrm{Y}_{\mathrm{p}}\right)=0, \forall \mathrm{X}_{\mathrm{p}} \in \mathrm{T}_{\mathrm{p}} \mathrm{M}\right\}
$$

is a distribution of rank 1 on $M$ [4. A complementary bundle of $T M^{\perp}$ in $T M$ is a rank $2 n-1$ non-degenerate distribution over $M$. It is called a screen distribution and is often denoted by $S(T M)$. In general, $S(T M)$ is not canonical (thus it is not unique). A lightlike hypersurface endowed with a specific screen distribution is denoted by the triple $(M, g, S(T M))$. As $\mathrm{TM}^{\perp}$ lies in the tangent bundle, the following result has an important role in studying the geometry of a lightlike hypersurface.

Theorem 2.1. [4] Let $(\mathrm{M}, \mathrm{g}, \mathrm{S}(\mathrm{TM}))$ be a lightlike hypersurface of $(\overline{\mathrm{M}}, \overline{\mathrm{g}})$. Then, there exists a unique vector bundle $\mathrm{N}(\mathrm{TM})$ of rank 1 over $\mathrm{M}$ such that for any non-zero section $\mathrm{E}$ of $\mathrm{TM}^{\perp}$ on a coordinate neighborhood $\mathcal{U} \subset \mathrm{M}$, there exists a unique section $\mathrm{N}$ of $\mathrm{N}(\mathrm{TM})$ on $\mathcal{U}$ satisfying $\overline{\mathrm{g}}(\mathrm{N}, \mathrm{E})=1$ and $\overline{\mathrm{g}}(\mathrm{N}, \mathrm{N})=\overline{\mathrm{g}}(\mathrm{N}, \mathrm{W})=0, \forall \mathrm{W} \in \Gamma(\mathrm{S}(\mathrm{TM}) \mid \mathcal{U})$.

Throughout the paper, all manifolds are supposed to be paracompact and smooth. We denote $\Gamma(\mathrm{E})$ the set of smooth sections of the vector bundle E. Also by $\perp$ and $\oplus$ we denote the orthogonal and nonorthogonal direct sum of two vector bundles. By Theorem 2.1 we may write down the following decompositions

$$
\begin{aligned}
\mathrm{TM} & =\mathrm{S}(\mathrm{TM}) \perp \mathrm{TM}^{\perp}, \\
\mathrm{T} \overline{\mathrm{M}} & =\mathrm{TM} \oplus \mathrm{N}(\mathrm{TM})=\mathrm{S}(\mathrm{TM}) \perp\left(\mathrm{TM}^{\perp} \oplus \mathrm{N}(\mathrm{TM})\right) .
\end{aligned}
$$

Let $\bar{\nabla}$ be the Levi-Civita connection on $(\bar{M}, \bar{g})$, then by using the second decomposition of (2.5) and considering a normalizing pair $\{\mathrm{E}, \mathrm{N}\}$ as in Theorem 2.1. we have the Gauss and Weingarten formulae in the form,

$$
\bar{\nabla}_{X} Y=\nabla_{X} Y+h(X, Y) \text {, and } \bar{\nabla}_{X} \mathrm{~V}=-A_{V} X+\nabla_{X}^{\perp} Y,
$$

for any $X, Y \in \Gamma\left(\left.T M\right|_{\mathcal{U}}\right), V \in \Gamma(N(T M))$, where $\nabla_{X} Y, A_{V} X \in \Gamma(T M)$ and $h(X, Y), \nabla_{X}^{\perp} V \in$ $\Gamma(\mathrm{N}(\mathrm{TM})) . \quad \nabla$ is an induced symmetric linear connection on $M, \nabla^{\perp}$ is a linear connection on the vector bundle $N(T M), h$ is a $\Gamma(N(T M))$-valued symmetric bilinear form and $A_{V}$ is the shape operator of $M$ concerning $V$.

Equivalently, consider a normalizing pair $\{\mathrm{E}, \mathrm{N}\}$ as in Theorem 2.1. Then (2.6) takes the form,

$$
\bar{\nabla}_{X} Y=\nabla_{X} Y+B(X, Y) N \text { and } \bar{\nabla}_{X} N=-A_{N} X+\tau(X) N,
$$

for any $X, Y \in \Gamma\left(\left.T M\right|_{\mathcal{U}}\right)$, where $B, A_{N}, \tau$ and $\nabla$ are called the local second fundamental form, the local shape operator, the transversal differential 1-form and the induced linear torsion free 
connection, respectively, on $\mathrm{TM}_{\mathcal{U}}$. It is important to mention that $\mathrm{B}$ is independent of the choice of screen distribution, in fact, from (2.7), we obtain $B(X, Y)=\bar{g}\left(\bar{\nabla}_{X} Y, E\right)$ and $\tau(X)=\bar{g}\left(\nabla_{X}^{\perp} N, E\right)$.

Let $\mathrm{P}$ be the projection morphism of TM on $\mathrm{S}(\mathrm{TM})$ with respect to the orthogonal decomposition of TM. We have,

$$
\nabla_{X} P Y=\nabla_{X}^{*} P Y+C(X, P Y) E, \text { and } \nabla_{X} E=-A_{E}^{*} X-\tau(X) E
$$

where $\nabla_{X}^{*} \mathrm{PY}$ and $A_{\mathrm{E}}^{*} \mathrm{X}$ belong to $\Gamma(\mathrm{S}(\mathrm{TM}))$. $\mathrm{C}, \mathrm{A}_{\mathrm{E}}^{*}$ and $\nabla^{*}$ are called the local second fundamental form, the local shape operator and the induced connection on $S(T M)$. The induced linear connection $\nabla$ is not a metric connection and we have, for any $\mathrm{X}, \mathrm{Y} \in \Gamma\left(\left.\mathrm{TM}\right|_{\mathcal{U}}\right)$,

$$
\left(\nabla_{X g}\right)(\mathrm{Y}, \mathrm{Z})=\mathrm{B}(\mathrm{X}, \mathrm{Y}) \theta(\mathrm{Z})+\mathrm{B}(\mathrm{X}, \mathrm{Z}) \theta(\mathrm{Y})
$$

where $\theta$ is a differential 1-form locally defined on $M$ by $\theta(\cdot):=\bar{g}(N, \cdot)$. Also, we have, $g\left(A_{E}^{*} X, P Y\right)=$ $\mathrm{B}(\mathrm{X}, \mathrm{PY}), \mathrm{g}\left(\mathrm{A}_{\mathrm{E}}^{*} \mathrm{X}, \mathrm{N}\right)=0, \mathrm{~B}(\mathrm{X}, \mathrm{E})=0$.

Using (2.7), the curvature tensor fields $\bar{R}$ and $R$ of $\bar{M}$ and $M$, respectively, are related as

$$
\begin{aligned}
& \overline{\mathrm{R}}(\mathrm{X}, \mathrm{Y}) \mathrm{Z}=\mathrm{R}(\mathrm{X}, \mathrm{Y}) \mathrm{Z}+\mathrm{B}(\mathrm{X}, \mathrm{Z}) \mathrm{A}_{\mathrm{N}} \mathrm{Y}-\mathrm{B}(\mathrm{Y}, \mathrm{Z}) \mathrm{A}_{\mathrm{N}} \mathrm{X} \\
& +\left\{\left(\nabla_{\mathrm{X}} \mathrm{B}\right)(\mathrm{Y}, \mathrm{Z})-\left(\nabla_{\mathrm{Y}} \mathrm{B}\right)(\mathrm{X}, \mathrm{Z})+\tau(\mathrm{X}) \mathrm{B}(\mathrm{Y}, \mathrm{Z})-\tau(\mathrm{Y}) \mathrm{B}(\mathrm{X}, \mathrm{Z})\right\} \mathrm{N}, \\
& \text { where }\left(\nabla_{\mathrm{X}} \mathrm{B}\right)(\mathrm{Y}, \mathrm{Z})=\mathrm{X} \cdot \mathrm{B}(\mathrm{Y}, \mathrm{Z})-\mathrm{B}\left(\nabla_{\mathrm{X}} \mathrm{Y}, \mathrm{Z}\right)-\mathrm{B}\left(\mathrm{Y}, \nabla_{\mathrm{X}} \mathrm{Z}\right) .
\end{aligned}
$$

\section{Lightlike hypersurfaces of indefinite Kenmotsu manifolds}

Let $(\bar{M}, \bar{\phi}, \xi, \eta, \bar{g})$ be an indefinite Kenmotsu manifold and $(M, g)$ be a lightlike hypersurface of $(\bar{M}, \bar{g})$, tangent to the structure vector field $\xi(\xi \in \mathrm{TM})$. If $\mathrm{E}$ is a local section of $\mathrm{TM}^{\perp}$, then $\overline{\mathrm{g}}(\bar{\phi} \mathrm{E}, \mathrm{E})=0$, and $\bar{\phi} \mathrm{E}$ is tangent to $M$. Thus $\bar{\phi}\left(\mathrm{TM}^{\perp}\right)$ is a distribution on $M$ of rank 1 such that $\bar{\phi}\left(T M^{\perp}\right) \cap T M^{\perp}=\{0\}$. This enables us to choose a screen distribution $S(T M)$ such that it contains $\bar{\phi}\left(\mathrm{TM}^{\perp}\right)$ as a vector subbundle. If we consider a local section $N$ of $N(T M)$, since $\bar{g}(\bar{\phi} N, E)=-\bar{g}(N, \bar{\phi} E)=0, \bar{\phi} E$ belongs to $S(T M)$. Since $\bar{g}(\bar{\phi} N, N)=0, \bar{\phi} N \in \Gamma(S(T M))$. From (2.1), we have $\bar{g}(\bar{\phi} N, \bar{\phi} E)=1$. Therefore, $\bar{\phi}\left(\mathrm{TM}^{\perp}\right) \oplus \bar{\phi}(\mathrm{N}(\mathrm{TM}))$ is a non-degenerate vector subbundle of $S(T M)$ of rank 2 . If $M$ is tangent to the structure vector field $\xi$, then, we may choose $S(T M)$ so that $\xi$ belongs to $S(T M)$. Using this and $\bar{g}(\bar{\phi} E, \xi)=\bar{g}(\bar{\phi} N, \xi)=0$, there exists a non-degenerate distribution $D_{0}$ of rank $2 n-4$ on $M$ such that

$$
\mathrm{S}(\mathrm{TM})=\left\{\bar{\phi}\left(\mathrm{TM}^{\perp}\right) \oplus \bar{\phi}(\mathrm{N}(\mathrm{TM}))\right\} \perp \mathrm{D}_{0} \perp<\xi>
$$

where $\langle\xi\rangle$ is the distribution spanned by $\xi$, that is, $\langle\xi\rangle=\operatorname{Span}\{\xi\}$. It is easy to check that the distribution $\mathrm{D}_{0}$ is invariant under $\bar{\phi}$, i.e. $\bar{\phi}\left(\mathrm{D}_{0}\right)=\mathrm{D}_{0}$.

Example 3.1. We consider the 7-dimensional manifold $\bar{M}^{7}=\left\{x \in \mathbb{R}^{7}: x_{7} \neq 0\right\}$, where $x=$ $\left(x_{1}, x_{2}, \ldots, x_{7}\right)$ are the standard coordinates in $\mathbb{R}^{7}$. The vector fields $e_{1}=x_{7} \frac{\partial}{\partial x_{1}}, e_{2}=x_{7} \frac{\partial}{\partial x_{2}}, e_{3}=$ $x_{7} \frac{\partial}{\partial x_{3}}, e_{4}=x_{7} \frac{\partial}{\partial x_{4}}, e_{5}=-x_{7} \frac{\partial}{\partial x_{5}}, e_{6}=-x_{7} \frac{\partial}{\partial x_{6}}, e_{7}=-x_{7} \frac{\partial}{\partial x_{7}}$ are linearly independent at each 
point of $\bar{M}^{7}$. Let $\bar{g}$ be the semi-Riemannian metric defined by $\bar{g}\left(e_{i}, e_{j}\right)=0, \forall i \neq j, i, j=1,2, \ldots, 7$ and $\bar{g}\left(e_{k}, e_{k}\right)=1, \forall k=1,2,3,4,7, \bar{g}\left(e_{m}, e_{m}\right)=-1, \forall m=5,6$. Let $\eta$ be the 1-form defined by $\eta(\cdot)=\bar{g}\left(\cdot, e_{7}\right)$. Let $\bar{\phi}$ be the $(1,1)$ tensor field defined by $\bar{\phi} e_{1}=-e_{2}, \bar{\phi} e_{2}=e_{1}, \bar{\phi} e_{3}=$ $-e_{4}, \bar{\phi} e_{4}=e_{3}, \bar{\phi} e_{5}=-e_{6}, \bar{\phi} e_{6}=e_{5}, \bar{\phi} e_{7}=0$. Then, using the linearity of $\bar{\phi}$ and $\bar{g}$, we have $\bar{\phi}^{2} \bar{X}=-\bar{X}+\eta(\bar{X}) e_{7}, \bar{g}(\bar{\phi} \bar{X}, \bar{\phi} \bar{Y})=\bar{g}(\bar{X}, \bar{Y})-\eta(\bar{X}) \eta(\bar{Y})$, for any $\bar{X}, \bar{Y} \in \Gamma\left(T \bar{M}^{7}\right)$. Thus, for $e_{7}=\xi,(\bar{\phi}, \xi, \eta, \bar{g})$ defines an almost contact metric structure on $\bar{M}^{7}$. Let $\bar{\nabla}$ be the LeviCivita connection with respect to the metric $\bar{g}$. Then, we have $\left[e_{i}, e_{7}\right]=e_{i}, \forall i=1,2, \ldots, 6$ and $\left[e_{i}, e_{j}\right]=0, \forall i \neq j, i, j=1,2, \ldots, 6$. The metric connection $\bar{\nabla}$ of the metric $\bar{g}$ is given by

$$
\begin{aligned}
2 \overline{\mathrm{g}}(\bar{\nabla} \overline{\mathrm{X}} \overline{\mathrm{Y}}, \overline{\mathrm{Z}})= & \overline{\mathrm{X}}(\overline{\mathrm{g}}(\overline{\mathrm{Y}}, \overline{\mathrm{Z}}))+\overline{\mathrm{Y}}(\overline{\mathrm{g}}(\overline{\mathrm{Z}}, \overline{\mathrm{X}}))-\overline{\mathrm{Z}}(\overline{\mathrm{g}}(\overline{\mathrm{X}}, \overline{\mathrm{Y}}))-\overline{\mathrm{g}}(\overline{\mathrm{X}},[\overline{\mathrm{Y}}, \overline{\mathrm{Z}}]) \\
& -\overline{\mathrm{g}}(\overline{\mathrm{Y}},[\overline{\mathrm{X}}, \overline{\mathrm{Z}}])+\overline{\mathrm{g}}(\overline{\mathrm{Z}},[\overline{\mathrm{X}}, \overline{\mathrm{Y}}])
\end{aligned}
$$

which is known as Koszul's formula. Using this formula, the non-vanishing covariant derivatives are given by $\bar{\nabla}_{e_{1}} e_{1}=-e_{7}, \bar{\nabla}_{e_{2}} e_{2}=-e_{7}, \bar{\nabla}_{e_{3}} e_{3}=-e_{7}, \bar{\nabla}_{e_{4}} e_{4}=-e_{7}, \bar{\nabla}_{e_{5}} e_{5}=e_{7}, \bar{\nabla}_{e_{6}} e_{6}=$ $e_{7}, \bar{\nabla}_{e_{1}} e_{7}=e_{1}, \bar{\nabla}_{e_{2}} e_{7}=e_{2}, \bar{\nabla}_{e_{3}} e_{7}=e_{3}, \bar{\nabla}_{e_{4}} e_{7}=e_{4}, \bar{\nabla}_{e_{5}} e_{7}=e_{5}, \bar{\nabla}_{e_{6}} e_{7}=e_{6}$. From these relations, it follows that the manifold $\bar{M}^{7}$ satisfies $\bar{\nabla}_{\bar{X}} \xi=\bar{X}-\eta(\bar{X}) \xi$. Hence, $\bar{M}^{7}$ is indefinite Kenmotsu manifold. We now define a hypersurface $M$ of $\left(\bar{M}^{7}, \bar{\phi}, \xi, \eta, \bar{g}\right)$ as $M=\left\{x \in \bar{M}^{7}: x_{5}=\right.$ $\left.x_{2}\right\}$. Thus, the tangent space $T M$ is spanned by $\left\{U_{i}\right\}_{1 \leq i \leq 6}$, where $U_{1}=e_{1}, U_{2}=e_{2}-e_{5}, U_{3}=$ $e_{3}, U_{4}=e_{4}, U_{5}=e_{6}, U_{6}=\xi$ and the 1-dimensional distribution $\mathrm{TM}^{\perp}$ of rank 1 is spanned by $E$, where $E=e_{2}-e_{5}$. It follows that $\mathrm{TM}^{\perp} \subset \mathrm{TM}$. Then $M$ is a 6-dimensional lightlike hypersurface of $\bar{M}^{7}$. Also, the transversal bundle $N(T M)$ is spanned by $N=\frac{1}{2}\left(e_{2}+e_{5}\right)$. Using the almost contact structure of $\bar{M}^{7}$ and (3.1), $D_{0}$ is spanned by $\{F, \bar{\phi} F\}$, where $F=U_{3}, \bar{\phi} F=-U_{4}$ and the distributions $\langle\xi\rangle, \bar{\phi}\left(\mathrm{TM}^{\perp}\right)$ and $\bar{\phi}(\mathrm{N}(\mathrm{TM}))$ are spanned, respectively, by $\xi, \bar{\phi} \mathrm{E}=\mathrm{U}_{1}+$ $\mathrm{U}_{5}$ and $\bar{\phi} \mathrm{N}=\frac{1}{2}\left(\mathrm{U}_{1}-\mathrm{U}_{5}\right)$. Hence, $\mathrm{M}$ is a lightlike hypersurface of $\overline{\mathrm{M}}^{7}$.

Moreover, from (2.5) and (3.1) we obtain the decompositions

$$
\begin{aligned}
& \mathrm{TM}=\left\{\bar{\phi}\left(\mathrm{TM}^{\perp}\right) \oplus \bar{\phi}(\mathrm{N}(\mathrm{TM}))\right\} \perp \mathrm{D}_{0} \perp<\xi>\perp \mathrm{TM}^{\perp}, \\
& \mathrm{T} \bar{M}=\left\{\bar{\phi}\left(\mathrm{TM}^{\perp}\right) \oplus \bar{\phi}(\mathrm{N}(\mathrm{TM}))\right\} \perp \mathrm{D}_{0} \perp<\xi>\perp\left(\mathrm{TM}^{\perp} \oplus \mathrm{N}(\mathrm{TM})\right) .
\end{aligned}
$$

Now, we consider the distributions on $M, D:=T^{\perp} \perp \bar{\phi}\left(\mathrm{TM}^{\perp}\right) \perp \mathrm{D}_{0}, \quad \mathrm{D}^{\prime}:=\bar{\phi}(\mathrm{N}(\mathrm{TM}))$. Then $\mathrm{D}$ is invariant under $\bar{\phi}$ and

$$
\mathrm{TM}=\mathrm{D} \oplus \mathrm{D}^{\prime} \perp\langle\xi\rangle .
$$

Let us consider the local lightlike vector fields $U:=-\bar{\phi} N, \quad V:=-\bar{\phi} E$. Then, from (3.4), any $X \in \Gamma(T M)$ is written as $X=R X+Q X+\eta(X) \xi, \quad Q X=\mathfrak{u}(X) U$, where $R$ and $Q$ are the projection morphisms of TM into $D$ and $D^{\prime}$, respectively, and $u$ is a differential 1-form locally defined on $M$ by $u(\cdot):=g(V, \cdot)$. Applying $\bar{\phi}$ to $X$ and using (2.1), one obtains $\bar{\phi} X=\phi X+u(X) N$, where $\phi$ is a tensor field of type $(1,1)$ defined on $M$ by $\phi X:=\bar{\phi} R X$. Also, we obtain

$$
\begin{aligned}
& \phi^{2} X=-X+\eta(X) \xi+u(X) u, \\
& \nabla_{X} \xi=X-\eta(X) \xi, \quad \forall X \in \Gamma(T M) .
\end{aligned}
$$


For the sake of future use, we have the following identities: for any $X, Y \in \Gamma(T M)$,

$$
\begin{aligned}
\mathrm{B}(\mathrm{X}, \xi) & =0 \\
\mathrm{C}(\mathrm{X}, \xi) & =\theta(\mathrm{X}) \\
\mathrm{B}(\mathrm{X}, \mathrm{U}) & =\mathrm{C}(\mathrm{X}, \mathrm{V}) \\
\left(\nabla_{X} \mathrm{u}\right) \mathrm{Y} & =-\mathrm{B}(\mathrm{X}, \phi \mathrm{Y})-\mathfrak{u}(\mathrm{Y}) \tau(\mathrm{X})-\eta(\mathrm{Y}) \mathrm{u}(\mathrm{X}) \\
\left(\nabla_{X} \phi\right) \mathrm{Y} & =\bar{g}(\bar{\phi} \mathrm{X}, \mathrm{Y}) \xi-\eta(\mathrm{Y}) \phi X-\mathrm{B}(\mathrm{X}, \mathrm{Y}) \mathrm{U}+\mathrm{u}(\mathrm{Y}) \mathrm{A}_{\mathrm{N}} \mathrm{X}
\end{aligned}
$$

Proposition 3.1. Let $\mathrm{M}$ be a lightlike hypersurface of an indefinite Kenmotsu manifold $\overline{\mathrm{M}}$ with $\xi \in \mathrm{TM}$. The Lie derivative of $\mathrm{g}$ with respect to the vector field $\mathrm{V}$ is given by, for any $\mathrm{X}, \mathrm{Y} \in \Gamma(\mathrm{TM})$,

$$
(\operatorname{Lvg})(X, Y)=X(u(Y))+Y(u(X))+u([X, Y])-2 u\left(\nabla_{X} Y\right)
$$

Proof: The proof follows by direct calculation.

The relation (3.12) can be rewritten as, for any $\mathrm{X}, \mathrm{Y} \in \Gamma(\mathrm{TM})$,

$$
\left(\operatorname{L}_{\vee} g\right)(X, Y)=\left(\nabla_{X} \mathfrak{u}\right) Y+\left(\nabla_{Y} \mathfrak{u}\right) X
$$

As the geometry of a lightlike hypersurface depends on the chosen screen distribution, it is important to investigate the relationship between geometrical objects induced by two screen distributions. We ask the following question: Is the Lie derivative $L_{V}(3.12)$ independent of the choice of a screen distribution $S(T M)$ ? The answer is negative because of the differential 1-form $\tau$ which appears in the relation (3.10) and which is not unique. Indeed, we prove the following with respect to a change in $S(T M)$.

Note that the 1-dimensional distribution $\mathrm{TM}^{\perp}$ is independent of the choice of a screen distribution and hence so is also $V:=-\bar{\phi} E\left(E \in \mathrm{TM}^{\perp}\right)$.

Suppose a screen $S(T M)$ changes to another screen $S(T M)^{\prime}$. Following are the transformation equations due to this change (see details in 4, page 87).

$$
\begin{aligned}
W_{i}^{\prime} & =\sum_{j=1}^{2 n-1} W_{i}^{j}\left(W_{j}-\epsilon_{j} c_{j} E\right), \\
N^{\prime} & =N-\frac{1}{2}\left\{\sum_{i=1}^{2 n-1} \epsilon_{i}\left(c_{i}\right)^{2}\right\} E+W, \\
\tau^{\prime}(X) & =\tau(X)+B(X, W), \\
\nabla_{X}^{\prime} Y & =\nabla_{X} Y+B(X, Y)\left\{\frac{1}{2}\left(\sum_{i=1}^{2 n-1} \epsilon_{i}\left(c_{i}\right)^{2}\right) E-W\right\},
\end{aligned}
$$

where $W=\sum_{i=1}^{2 n-1} c_{i} W_{i},\left\{W_{i}\right\}$ and $\left\{W_{i}^{\prime}\right\}$ are the local orthonormal bases of $S(T M)$ and $S(T M)^{\prime}$ with respective transversal sections $N$ and $N^{\prime}$ for the same null section $E$. Here $c_{i}$ and $W_{i}^{j}$ are smooth functions on $\mathcal{U}$ and $\left\{\epsilon_{1}, \ldots, \epsilon_{2 n-1}\right\}$ is the signature of the basis $\left\{W_{1}, \ldots, W_{2 n-1}\right\}$. The Lie 
derivatives $L_{V}$ and $L_{V}^{\prime}$ of the screen distributions $S(T M)$ and $S(T M)^{\prime}$, respectively, are related through the relation [12]:

$$
\begin{aligned}
& \left(\mathrm{L}_{V}^{\prime} \mathrm{g}\right)(\mathrm{X}, \mathrm{Y})=\left(\nabla_{X}^{\prime} \mathrm{u}\right) \mathrm{Y}+\left(\nabla_{Y}^{\prime} \mathrm{u}\right) \mathrm{X} \\
& =-\mathrm{B}(\mathrm{X}, \phi \mathrm{Y})-\mathfrak{u}(\mathrm{Y}) \tau^{\prime}(\mathrm{X})-\eta(\mathrm{Y}) \mathrm{u}(\mathrm{X})-\mathrm{B}(\mathrm{Y}, \phi X) \\
& -\mathfrak{u}(X) \tau^{\prime}(Y)-\eta(X) \mathfrak{u}(Y) \\
& =-\mathrm{B}(\mathrm{X}, \phi \mathrm{Y})-\mathfrak{u}(\mathrm{Y})(\tau(X)+\mathrm{B}(\mathrm{X}, \mathrm{W}))-\mathfrak{\eta}(\mathrm{Y}) \mathfrak{u}(\mathrm{X}) \\
& -\mathrm{B}(\mathrm{Y}, \phi X)-\mathfrak{u}(\mathrm{X})(\tau(\mathrm{Y})+\mathrm{B}(\mathrm{Y}, \mathrm{W}))-\eta(\mathrm{X}) \mathfrak{u}(\mathrm{Y}) \\
& =\left(L_{V} g\right)(X, Y)-\mathfrak{u}(X) B(Y, W)-\mathfrak{u}(Y) B(X, W) \text {. }
\end{aligned}
$$

The Lie derivative $L_{V}$ is unique, that is, $L_{V}$ is independent of $S(T M)$, if and only if, the second fundamental form $h$ (or equivalently B) of $M$ vanishes identically on $M$.

If a $(2 n+1)$-dimensional Kenmotsu manifold $\bar{M}$ has a constant $\bar{\phi}$-sectional curvature $c$, then the Ricci tensor $\overline{\text { Ric }}$ and the scalar curvature $\overline{\mathrm{r}}$ are given by 9 ]

$$
\overline{\operatorname{Ric}}=\frac{1}{2}(n(c-3)+c+1) \bar{g}-\frac{1}{2}(n+1)(c+1) \eta \otimes \eta .
$$

This means that $\bar{M}$ is $\eta$-Einstein. Since $\bar{M}$ is Kenmotsu and $\eta$-Einstein, by Corollary 9 in $[9, \bar{M}$ is an Einstein one and consequently, $c+1=0$, that is, $c=-1$. So, the Ricci tensor (3.16) becomes $\overline{\text { Ric }}=-2 n \bar{g}$ and the scalar curvature is given by $\bar{r}=-2 n(2 n+1)$.

Thus, if a Kenmotsu manifold $\bar{M}$ is a space form, then it is Einstein and $\mathrm{c}=-1$.

Let $M$ be a lightlike hypersurface of an indefinite Kenmotsu space form $\bar{M}(c)$ with $\xi \in T M$. Using (3.7) and the fact that $B(., E)=0$, the local second fundamental form $B$ of $M$ can be written as, for any $\mathrm{X}, \mathrm{Y} \in \Gamma(\mathrm{TM})$,

$$
B(X, Y)=\sum_{i=1}^{2 n-4} \frac{B\left(X, F_{i}\right)}{g\left(F_{i}, F_{i}\right)} g\left(Y, F_{i}\right)+v(Y) u\left(A_{E}^{*} X\right)+u(Y) v\left(A_{E}^{*} X\right),
$$

where $\left\{F_{i}\right\}_{1 \leq i \leq 2 n-4}$ is an orthogonal basis of $D_{0}$ and $g\left(F_{i}, F_{i}\right) \neq 0$. This means that $M$ is not totally geodesic in general. So, only some privileged conditions on distributions could allow $M$ to be totally geodesic.

Next we study some classes of lightlike hypersurfaces $M$ of $\bar{M}(\mathrm{c})$, tangent to the structure vector field $\xi$, which are totally geodesic. Let us consider the pair $\{\mathrm{E}, \mathrm{N}\}$ on $\mathcal{U} \subset \mathrm{M}$ (see Theorem 2.1) and by using (2.10), we obtain

$$
\left(\nabla_{X} \mathrm{~B}\right)(\mathrm{Y}, \mathrm{Z})-\left(\nabla_{\mathrm{Y}} \mathrm{B}\right)(\mathrm{X}, \mathrm{Z})=\tau(\mathrm{Y}) \mathrm{B}(\mathrm{X}, \mathrm{Z})-\tau(\mathrm{X}) \mathrm{B}(\mathrm{Y}, \mathrm{Z}) .
$$

Theorem 3.1. Let $M$ be a lightlike hypersurface of an indefinite Kenmotsu space form $\bar{M}(\mathrm{c})$, with $\xi \in \mathrm{TM}$. Then, the Lie derivative of the second fundamental form $\mathrm{B}$ with respect to $\xi$ is given by

$$
\left(\mathrm{L}_{\xi} \mathrm{B}\right)(\mathrm{X}, \mathrm{Y})=(1-\tau(\xi)) \mathrm{B}(\mathrm{X}, \mathrm{Y}), \forall \mathrm{X}, \mathrm{Y} \in \Gamma(\mathrm{TM}) .
$$


Moreover, if $\tau(\xi) \neq 1$, then $\xi$ is a Killing vector field with respect to the second fundamental form $\mathrm{B}$ if and only if $\mathrm{M}$ is totally geodesic.

Proof: Using (2.11), we obtain

$$
\left(\nabla_{\xi} \mathrm{B}\right)(\mathrm{X}, \mathrm{Y})=\left(\mathrm{L}_{\xi} \mathrm{B}\right)(\mathrm{X}, \mathrm{Y})-2 \mathrm{~B}(\mathrm{X}, \mathrm{Y}) .
$$

Likewise, Using again (2.11), we have

$$
\left(\nabla_{X} \mathrm{~B}\right)(\xi, Y)=-\mathrm{B}(\mathrm{X}, \mathrm{Y}) .
$$

Subtracting (3.20) and (3.21), we obtain

$$
\left(\nabla_{\xi} \mathrm{B}\right)(\mathrm{X}, \mathrm{Y})-\left(\nabla_{\mathrm{X}} \mathrm{B}\right)(\xi, \mathrm{Y})=\left(\mathrm{L}_{\xi} \mathrm{B}\right)(\mathrm{X}, \mathrm{Y})-\mathrm{B}(\mathrm{X}, \mathrm{Y}) .
$$

From (3.18) and after calculations, the left hand side of (3.22) becomes

$$
\left(\nabla_{\xi} \mathrm{B}\right)(\mathrm{X}, \mathrm{Y})-\left(\nabla_{\mathrm{X}} \mathrm{B}\right)(\xi, \mathrm{Y})=-\tau(\xi) \mathrm{B}(\mathrm{X}, \mathrm{Y}) .
$$

The expressions (3.22) and (3.23) implies $\left(\mathrm{L}_{\xi} \mathrm{B}\right)(\mathrm{X}, \mathrm{Y})=(1-\tau(\xi)) \mathrm{B}(\mathrm{X}, \mathrm{Y})$. The last assertion is obvious by definitions of Killing distribution and totally geodesic submanifold.

As an example to the last part of the Theorem 3.1, we have a lightlike hypersurface of an indefinite Kenmotsu space form, tangent to the structure vector field $\xi$, with parallel vector field $\mathrm{U}$ or $\mathrm{V}$. In fact, when the vector field $\mathrm{U}$ or $\mathrm{V}$ is parallel, the differential 1-form $\tau$ vanishes on $\mathrm{M}$ and consequently, the equivalence of the Theorem 3.1 holds.

The second fundamental form $h=B \otimes N$ of $M$ is said to be parallel if $(\nabla \times h)(Y, Z)=$ $0, \forall X, Y, Z \in \Gamma(T M)$. That is,

$$
\left(\nabla_{X} \mathrm{~B}\right)(\mathrm{Y}, \mathrm{Z})=-\tau(\mathrm{X}) \mathrm{B}(\mathrm{Y}, \mathrm{Z}) .
$$

Theorem 3.2. Let $\mathrm{M}$ be a lightlike hypersurface of an indefinite Kenmotsu space form $\bar{M}(\mathrm{c})$ with $\xi \in \mathrm{TM}$. If the second fundamental form $\mathrm{h}$ of $\mathrm{M}$ is parallel, then $\mathrm{M}$ is totally geodesic.

Proof: Suppose that the second fundamental form $h$ of $M$ is parallel. Using (3.24), we obtain

$$
\left(\nabla_{\xi} \mathrm{B}\right)(\mathrm{X}, \mathrm{Y})=-\tau(\xi) \mathrm{B}(\mathrm{X}, \mathrm{Y}) .
$$

From (2.11) and using (3.19), the left hand side of (3.25) becomes

$$
\left(\nabla_{\xi} \mathrm{B}\right)(\mathrm{X}, \mathrm{Y})=\left(\mathrm{L}_{\xi} \mathrm{B}\right)(\mathrm{X}, \mathrm{Y})-2 \mathrm{~B}(\mathrm{X}, \mathrm{Y})=-(1+\tau(\xi)) \mathrm{B}(\mathrm{X}, \mathrm{Y}) .
$$

From the expressions (3.25) and (3.26) we complete the proof.

This means that any parallel lightlike hypersurface $M$ of an indefinite Kenmotsu manifold $\bar{M}$ admits a metric connection. 
The covariant derivative of the second fundamental form $h$ depends on $\nabla, N$ and $\tau$ which depend on the choice of the screen vector bundle. Using equations (3.14), the covariant derivatives $\nabla$ of $h=B \otimes N$ and $\nabla^{\prime}$ of $h^{\prime}=B \otimes N^{\prime}$ in the screen distributions $S(T M)$ and $S(T M)^{\prime}$, respectively, are related as follows: for any $\mathrm{X}, \mathrm{Y}, \mathrm{Z} \in \Gamma(\mathrm{TM})$,

$$
\bar{g}\left(\left(\nabla_{X}^{\prime} h^{\prime}\right)(Y, Z), E\right)=\bar{g}\left(\left(\nabla_{X} h\right)(Y, Z), E\right)+\mathcal{L}_{(X, Y)} Z,
$$

with $\mathcal{L}_{(X, Y)} Z=B(X, Y) B(Z, W)+B(X, Z) B(Y, W)+B(Y, Z) B(X, W)$. It is easy to check that the parallelism of $h$ is independent of the screen distribution $S(T M)\left(\nabla^{\prime} h^{\prime} \equiv \nabla h\right)$ if and only the second fundamental form $B$ of $M$ vanishes identically on $M$.

We note that the Theorem 3.2 arises when the local second fundamental form $B$ of $M$ is also parallel. Therefore, the Theorem 3.2 generates some lightlike geometric aspects on any parallel lightlike hypersurface of an indefinite Kenmotsu manifold by using the Theorem 2.2 in [4].

From (2.3) and (2.10), a direct calculation shows that

$$
\begin{aligned}
& \left(\nabla_{X} C\right)(Y, P Z)-\left(\nabla_{Y} C\right)(X, P Z)+\tau(Y) C(X, P Z)-\tau(X) C(Y, P Z) \\
& =\bar{g}(X, P Z) \theta(Y)-\bar{g}(Y, P Z) \theta(X) .
\end{aligned}
$$

Lemma 3.1. Let $(\mathrm{M}, \mathrm{g}, \mathrm{S}(\mathrm{TM}))$ be a lightlike hypersurface of an indefinite Kenmotsu manifold $(\overline{\mathrm{M}}, \overline{\mathrm{g}})$ with $\xi \in \mathrm{TM}$. Then, the covariant derivative of $v$ and the Lie derivative of $\mathrm{g}$ with respect to the vector field $\mathrm{U}$ are given, respectively, by, for any $\mathrm{X}, \mathrm{Y} \in \Gamma(\mathrm{TM})$,

$$
\begin{aligned}
\left(\nabla_{\mathrm{X} v}\right) \mathrm{Y} & =-\mathrm{C}(\mathrm{X}, \phi \mathrm{Y})-v(\mathrm{X}) \eta(\mathrm{Y})+\tau(\mathrm{X}) v(\mathrm{Y}) \\
\left(\mathrm{Lug}_{\mathrm{u}}\right)(\mathrm{X}, \mathrm{Y}) & =\mathrm{X}(v(\mathrm{Y}))+\mathrm{Y}(v(\mathrm{X}))+v([\mathrm{X}, \mathrm{Y}])-2 v\left(\nabla_{\mathrm{X}} \mathrm{Y}\right)
\end{aligned}
$$

where $v(\cdot):=\mathrm{g}(\mathrm{u}, \cdot)$.

Proof: The proof of (3.28) and (3.29) follows from direct calculations.

The Lie derivative (3.29) can be written in terms of the second fundamental form C of S(TM) using the relation

$$
v\left(\nabla_{\mathrm{X}} \mathrm{Y}\right)=\mathrm{C}(\mathrm{X}, \phi \mathrm{Y})+\eta(\mathrm{Y}) v(\mathrm{X}), \quad \forall \mathrm{X}, \mathrm{Y} \in \Gamma(\mathrm{TM})
$$

Example 3.2. Let $M$ be a hypersurface of $\bar{M}^{7}$ defined in the example 3.1] The tangent space TM is spanned by $\left\{U_{i}\right\}_{1 \leq i \leq 6}$, where $U_{1}=e_{1}, U_{2}=e_{2}-e_{5}, U_{3}=e_{3}, U_{4}=e_{4}, U_{5}=e_{6}, U_{6}=\xi$ and the 1-dimensional distribution $\mathrm{TM}^{\perp}$ of rank 1 is spanned by $\mathrm{E}$, where $\mathrm{E}=e_{2}-e_{5}$. Also, the transversal bundle $\mathrm{N}(\mathrm{TM})$ is spanned by $\mathrm{N}=\frac{1}{2}\left(e_{2}+e_{5}\right)$. It follows that $\mathrm{TM}^{\perp} \subset \mathrm{TM}$. Then $M$ is a 6-dimensional lightlike hypersurface of $\bar{M}^{7}$ having a local quasi-orthogonal field of frames $\left\{\mathrm{U}_{1}, \mathrm{U}_{2}=\mathrm{E}, \mathrm{U}_{3}, \mathrm{U}_{4}, \mathrm{U}_{5}, \mathrm{U}_{6}=\xi, \mathrm{N}\right\}$ along $M$. Denote by $\bar{\nabla}$ the Levi-Civita connection on $\overline{\mathrm{M}}^{7}$. Then, by straightforward calculations, we obtain $\bar{\nabla}_{X} N=0, \forall X \in \Gamma(T M)$. Using these equations above, the differential 1-form $\tau$ vanishes i.e. $\tau(X)=0$, for any $X \in \Gamma(T M)$. So, from the Gauss and Weingarten formulae we have $A_{N} X=0, A_{E}^{*} X=0$ and $\nabla_{X} E=0, \forall X \in \Gamma(T M)$. Therefore, by 
Theorem 2.2 and Proposition 2.7 in [4] pages 88-89, the lightlike hypersurface $M$ of $\bar{M}^{7}$ is totally geodesic and its distribution is parallel. The non-vanishing components of the Lie derivatives (3.12) and (3.29) are given by

$$
\begin{aligned}
& \operatorname{Lvg}_{v}\left(U_{1}, \xi\right)=\operatorname{Lvg}\left(\xi, U_{1}\right)=1, \quad \operatorname{Lvg}\left(U_{5}, \xi\right)=\operatorname{Lvg}\left(\xi, U_{5}\right)=-1, \\
& \operatorname{Lvg}(U, \xi)=\operatorname{Lvg}(\xi, U)=-1, \quad \operatorname{Lug}(V, \xi)=\operatorname{Lug}(\xi, V)=-1, \\
& \operatorname{Lug}\left(U_{1}, \xi\right)=\operatorname{Lug}\left(\xi, U_{1}\right)=\frac{1}{2}, \quad \operatorname{Lug}\left(U_{5}, \xi\right)=\operatorname{Lug}\left(\xi, U_{5}\right)=-\frac{1}{2} .
\end{aligned}
$$

\section{Lightlike Geometry of Leaves in Indefinite Kenmotsu Man- ifolds}

Let $M$ be a lightlike hypersurface of an indefinite Kenmotsu space form $\bar{M}(c)$ with $\xi \in$ TM. From the differential geometry of lightlike hypersurfaces, we recall the following desirable property for lightlike geometry. It is known that lightlike submanifolds whose screen distribution is integrable have interesting properties. Now, we study the geometry of leaves of integrable distributions with specific attention to leaves of screen distribution $\mathrm{S}(\mathrm{TM})$, the distributions $\mathrm{D}, \mathrm{D}_{0}, \mathrm{D}_{0} \perp\langle\xi\rangle$ and $\left\{\bar{\phi}\left(\mathrm{TM}^{\perp}\right) \oplus \bar{\phi}(\mathrm{N}(\mathrm{TM}))\right\} \perp \mathrm{D}_{0}$. By Theorem 2.3 in [4 page 89, the screen distribution $\mathrm{S}(\mathrm{TM})$ of $M$ is integrable if and only if the second fundamental form of $S(T M)$ is symmetric on $\Gamma(S(T M))$. However, for any $X, Y \in \Gamma(D \perp\langle\xi\rangle), \mathfrak{u}([X, Y])=B(X, \phi Y)-B(\phi X, Y)$. So, it is very easy to see that the distribution $\mathrm{D} \perp\langle\xi\rangle$ is integrable if and only if $\mathrm{B}(\mathrm{X}, \phi \mathrm{Y})=\mathrm{B}(\phi X, \mathrm{Y})$.

Theorem 4.1. Let $(\mathrm{M}, \mathrm{g}, \mathrm{S}(\mathrm{TM}))$ be a lightlike hypersurface of an indefinite Kenmotsu space form $\overline{\mathrm{M}}(\mathrm{c})$ with $\xi \in \mathrm{TM}$ such that the distribution $\mathrm{D} \perp\langle\xi\rangle$ is integrable. Then, $\mathrm{M}$ is $\mathrm{D} \perp\langle\xi\rangle$-totally geodesic if and only if $\bar{\phi}\left(\mathrm{TM}^{\perp}\right)$ is a $\mathrm{D} \perp\langle\xi\rangle$-Killing distribution.

Proof: Since D $\perp\langle\xi\rangle$ is integrable, using (3.10) and (3.12), one obtains,

$$
\left(L_{V} g\right)(X, Y)=-B(X, \phi Y)-B(\phi X, Y)=-2 B(X, \phi Y), \quad X, Y \in \Gamma(D \perp\langle\xi\rangle) .
$$

Using (3.7) and the fact that $\bar{\phi}(\mathrm{D} \perp\langle\xi\rangle)=\mathrm{D}$, we complete the proof.

Note that the Theorem 4.1 also holds when the distribution $\mathrm{D} \perp\langle\xi\rangle$ is replaced by $\mathrm{D}$.

Example 4.1. Consider the lightlike hypersurface $M$ of $\bar{M}^{7}$ defined in the example 3.2, Since $M$ is totally geodesic, so it is obviously $\mathrm{D} \perp\langle\xi\rangle$-totally geodesic. Since the only nonvanishing brackets on the distribution $\mathrm{D} \perp\langle\xi\rangle$ are $[\mathrm{V}, \xi]=\mathrm{V},[\mathrm{E}, \xi]=\mathrm{E},[\mathrm{F}, \xi]=\mathrm{F}$ and $[\bar{\phi} \mathrm{F}, \xi]=\bar{\phi} \mathrm{F}$, it is easy to check that the distribution $D \perp\langle\xi\rangle$ is integrable and $\left(L_{V} g\right)(X, Y)=-2 B(X, \phi Y)=0$, $\mathrm{X}, \mathrm{Y} \in \Gamma(\mathrm{D} \perp\langle\xi\rangle)$, that is, $\bar{\phi}\left(\mathrm{TM}^{\perp}\right)$ is a $\mathrm{D} \perp\langle\xi\rangle$-Killing distribution.

Proposition 4.1. Let $(\mathrm{M}, \mathrm{g}, \mathrm{S}(\mathrm{TM}))$ be a lightlike hypersurface of an indefinite Kenmotsu space form $\bar{M}(\mathrm{c})$ with $\xi \in \mathrm{TM}$. If the screen distribution $\mathrm{S}(\mathrm{TM})$ is integrable, then,

$$
\left(L_{\xi} C\right)(X, P Y)=\tau(\xi) C(X, P Y), \quad X, Y \in \Gamma(T M) .
$$


Proof: If the screen distribution $S(T M)$ of a lightlike hypersurface $M$ is integrable, then, from (3.27) and using (3.8), we have, for any $X, Y \in \Gamma(T M)$,

$$
\left(\nabla_{\xi} \mathrm{C}\right)(\mathrm{X}, \mathrm{PY})-\left(\nabla_{\mathrm{X}} \mathrm{C}\right)(\xi, \mathrm{PY})=\eta(\mathrm{PY}) \theta(\mathrm{X})+\tau(\xi) \mathrm{C}(\mathrm{X}, \mathrm{PY})
$$

On the other hand, using (3.8), we have

$$
\begin{aligned}
\left(\nabla_{\xi} \mathrm{C}\right)(\mathrm{X}, \mathrm{PY}) & =\xi(\mathrm{C}(\mathrm{X}, \mathrm{PY}))-\mathrm{C}\left(\nabla_{\xi} \mathrm{X}, \mathrm{PY}\right)-\mathrm{C}\left(\mathrm{X}, \nabla_{\xi}(\mathrm{PY})\right) \\
& =\left(\mathrm{L}_{\xi} \mathrm{C}\right)(\mathrm{X}, \mathrm{PY})-2 \mathrm{C}(\mathrm{X}, \mathrm{PY})+\eta(\mathrm{PY}) \theta(\mathrm{X}) \\
\text { and }\left(\nabla_{\mathrm{X}} \mathrm{C}\right)(\xi, \mathrm{PY}) & =\mathrm{X}(\mathrm{C}(\xi, \mathrm{PY}))-\mathrm{C}\left(\nabla_{\mathrm{X}} \xi, \mathrm{PY}\right)-\mathrm{C}\left(\xi, \nabla_{X} \mathrm{PY}\right) \\
& =-2 \mathrm{C}(\mathrm{X}, \mathrm{PY}) .
\end{aligned}
$$

Putting (4.3) and (4.4) together in (4.2), we obtain (4.1).

Let us assume that the screen distribution $S(T M)$ of $M$ is integrable and let $M^{\prime}$ be a leaf of $\mathrm{S}(\mathrm{TM})$. Then, using (2.7) and (2.8), we obtain, for any $\mathrm{X}, \mathrm{Y} \in \Gamma\left(\mathrm{TM}^{\prime}\right)$,

$$
\bar{\nabla}_{X} Y=\nabla_{X}^{*} Y+C(X, Y) E+B(X, Y) N=\nabla_{X}^{\prime} Y+h^{\prime}(X, Y)
$$

where $\nabla^{\prime}$ and $h^{\prime}$ are, respectively, the Levi-Civita connection and second fundamental form of $M^{\prime}$ in $\bar{M}$. Thus, for any $\mathrm{X}, \mathrm{Y} \in \Gamma\left(\mathrm{TM}^{\prime}\right)$,

$$
h^{\prime}(X, Y)=C(X, Y) E+B(X, Y) N
$$

In the sequel, we need the following lemma.

Lemma 4.1. Let (M, g, S(TM)) be a screen integrable lightlike hypersurface of an indefinite Kenmotsu manifold $(\overline{\mathrm{M}}, \overline{\mathrm{g}})$ with $\xi \in \mathrm{TM}$ and $\mathrm{M}^{\prime}$ be a leaf of $\mathrm{S}(\mathrm{TM})$. Then,

$$
\begin{aligned}
\nabla_{X}^{\prime} \xi & =X-\eta(X) \xi, \\
\nabla_{X}^{\prime} \mathrm{u} & =-v(X) \xi-v\left(A_{N} X\right) E-v\left(A_{E}^{*} X\right) N+\bar{\phi}\left(A_{N} X\right)+\tau(X) U, \\
\nabla_{X}^{\prime} V & =-u(X) \xi-u\left(A_{N} X\right) E-u\left(A_{E}^{*} X\right) N+\bar{\phi}\left(A_{E}^{*} X\right)-\tau(X) V,
\end{aligned}
$$

for any $\mathrm{X} \in \Gamma\left(\mathrm{TM}^{\prime}\right)$.

Proof: From a straightforward calculation we complete the proof.

It is well known that the second fundamental form and the shape operators of a non-degenerate hypersurface (in general, submanifold) are related by means of the metric tensor field. Contrary to this, we see from (2.8), in the case of lightlike hypersurfaces, the second fundamental forms on $M$ and their screen distribution $S(T M)$ are related to their respective shape operators $A_{N}$ and $A_{E}^{*}$. As the shape operator is an information tool in studying the geometry of submanifolds, their studying turns out very important. For instance, in [5] a class of lightlike hypersurfaces whose shape operators are the same as the one of their screen distribution up to a conformal non zero 
smooth factor in $\mathcal{F}(M)$ was considered. That work gave a way to generate, under some geometric conditions, an integrable canonical screen (see [5] for more details).

Next, we study these operators and give their implications in lightlike hypersurface of indefinite Kenmotsu manifolds with $\xi \in \mathrm{TM}$.

Proposition 4.2. Let $(M, g, S(T M))$ be a screen integrable lightlike hypersurface of an indefinite Kenmotsu manifold $(\overline{\mathrm{M}}, \overline{\mathrm{g}})$ with $\xi \in \mathrm{TM}$ and $\mathrm{M}^{\prime}$ be a leaf of $\mathrm{S}(\mathrm{TM})$. Then we have

(i) The vector field $\mathrm{U}$ is parallel with respect to the Levi-Civita connection $\nabla^{\prime}$ on $\mathrm{M}^{\prime}$ if and only if

$$
A_{N} X=u\left(A_{N} X\right) U, \quad \forall X \in \Gamma\left(T M^{\prime}\right),
$$

$v$ and $\tau$ vanish on $\mathrm{M}^{\prime}$.

(ii) The vector field $\mathrm{V}$ is parallel with respect to the Levi-Civita connection $\nabla^{\prime}$ on $\mathrm{M}^{\prime}$ if and only if

$$
A_{\mathrm{E}}^{*} \mathrm{X}=v\left(\mathrm{~A}_{\mathrm{E}}^{*} \mathrm{X}\right) \mathrm{V}, \quad \forall \mathrm{X} \in \Gamma\left(\mathrm{TM}^{\prime}\right)
$$

$u$ and $\tau$ vanishes on $\mathrm{M}^{\prime}$.

Proof: (i) Suppose $\mathrm{U}$ is parallel with respect to the Levi-Civita connection $\nabla^{\prime}$ on $M^{\prime}$. Then, by using (4.8), we have, for any $\mathrm{X} \in \Gamma\left(\mathrm{TM}^{\prime}\right)$,

$$
\bar{\phi}\left(A_{N} X\right)=v(X) \xi+v\left(A_{N} X\right) E+v\left(A_{E}^{*} X\right) N-\tau(X) U
$$

Since $\bar{\phi}\left(A_{N} X\right)=\phi\left(A_{N} X\right)+u\left(A_{N} X\right) N$, by using (3.9), we obtain

$$
\phi\left(A_{N} X\right)=v(X) \xi+v\left(A_{N} X\right) E-\tau(X) U
$$

Apply $\phi$ to (4.11) and using (3.5) and the fact that $\phi \mathrm{U}=0$, we obtain

$$
\begin{aligned}
A_{N} X & =\eta\left(A_{N} X\right) \xi+u\left(A_{N} X\right) u+v\left(A_{N} X\right) v \\
& =u\left(A_{N} X\right) u+v\left(A_{N} X\right) v
\end{aligned}
$$

since $\theta(X)=0$, for any $X \in \Gamma\left(\mathrm{TM}^{\prime}\right)$. Putting (4.12) in (4.8) and using (3.9), one obtains $v(X) \xi-$ $\tau(X) U=0$ which is equivalent to $v(X)=0$ and $\tau(X)=0$. Since $A_{N} X \in \Gamma\left(T M^{\prime}\right)$, then (4.12) is reduced to $A_{N} X=u\left(A_{N} X\right) U$. The converse is obvious. In the similar way, by using (4.9) the assertion (ii) follows.

Corollary 4.1. (to Proposition 4.2) Let (M, g, S(TM)) be a screen integrable lightlike hypersurface of an indefinite Kenmotsu manifold $(\overline{\mathrm{M}}, \overline{\mathrm{g}})$ with $\xi \in \mathrm{TM}$ and $\mathrm{M}^{\prime}$ be a leaf of $\mathrm{S}(\mathrm{TM})$ such $\mathrm{U}$ and $\mathrm{V}$ are parallel with respect to the Levi-Civita connection $\nabla^{\prime}$ on $\mathrm{M}^{\prime}$. Then, the type number $\mathrm{t}^{\prime}(\mathrm{x})$ of $\mathrm{M}^{\prime}$ (with $\mathrm{x} \in \mathrm{M}^{\prime}$ ) satisfies $\mathrm{t}^{\prime}(\mathrm{x}) \leq 1$. 
Proof: The proof follows from Proposition 4.2 ,

Let $W$ be an element of $\bar{\phi}\left(\mathrm{TM}^{\perp}\right) \oplus \bar{\phi}(\mathrm{N}(\mathrm{TM}))$ which is a non-degenerate vector subbundle of $\mathrm{S}(\mathrm{TM})$ of rank 2 . Then there exist non-zero functions $\mathrm{a}$ and $\mathrm{b}$ such that

$$
\mathrm{K}=\mathrm{aV}+\mathrm{bu}
$$

It is easy to check that $a=v(K)$ and $b=u(K)$. Let $\mathrm{K}$ be a 1 -form locally defined by $\mathrm{k}(\cdot)=\mathrm{g}(\mathrm{K}, \cdot)$.

Lemma 4.2. Let $(\mathrm{M}, \mathrm{g}, \mathrm{S}(\mathrm{TM}))$ be a lightlike hypersurface of an indefinite Kenmotsu manifold $(\overline{\mathrm{M}}, \overline{\mathrm{g}})$ with $\xi \in \mathrm{TM}$. Then, the covariant derivative of $\mathrm{\kappa}$ and the Lie derivative of $\mathrm{g}$ with respect to the vector field $\mathrm{K}$ are given, respectively, by

$$
\begin{aligned}
\left(\nabla_{X} \mathrm{~K}\right) \mathrm{Y} & =-v(\mathrm{~K}) \mathrm{B}(\mathrm{X}, \phi \mathrm{Y})-\mathrm{u}(\mathrm{K}) \mathrm{C}(\mathrm{X}, \phi \mathrm{Y})-\mathrm{\kappa}(\mathrm{X}) \eta(\mathrm{Y}) \\
\left(\mathrm{L}_{\mathrm{K}} \mathrm{g}\right)(\mathrm{X}, \mathrm{Y}) & =\mathrm{X}(\mathrm{\kappa}(\mathrm{Y}))+\mathrm{Y}(\mathrm{\kappa}(\mathrm{X}))+\kappa([\mathrm{X}, \mathrm{Y}])-2 \kappa\left(\nabla_{X} \mathrm{Y}\right)
\end{aligned}
$$

for any $\mathrm{X}, \mathrm{Y} \in \Gamma(\mathrm{TM})$.

Proof: Using (3.10) and (3.28), we obtain, for any $\mathrm{X}, \mathrm{Y} \in \Gamma(\mathrm{TM})$,

$$
\left(\nabla_{X K}\right) Y=-v(K) B(X, \phi Y)-u(K) C(X, \phi Y)-\kappa(X) \eta(Y)
$$

which proves (4.14) and (4.15) follows from a direct calculation.

From (3.30), one obtains, for any $\mathrm{X}, \mathrm{Y} \in \Gamma(\mathrm{TM})$,

$$
\kappa\left(\nabla_{X} Y\right)=v(K) B(X, \phi Y)+u(K) C(X, \phi Y)+\kappa(X) \eta(Y)
$$

Lemma 4.3. Let $(\mathrm{M}, \mathrm{g}, \mathrm{S}(\mathrm{TM}))$ be a screen integrable lightlike hypersurface of an indefinite Kenmotsu manifold $(\overline{\mathrm{M}}, \overline{\mathrm{g}})$ with $\xi \in \mathrm{TM}$ and $\mathrm{M}^{\prime}$ be a leaf of $\mathrm{S}(\mathrm{TM})$. Then, for any, $\mathrm{X}, \mathrm{Y} \in \Gamma\left(\mathrm{TM}^{\prime}\right)$,

$$
\begin{aligned}
\kappa\left(\nabla_{X}^{\prime} Y\right) & =-\kappa\left(\bar{\phi} h^{\prime}(X, \phi Y)\right)+\kappa(X) \eta(Y) \\
\kappa([X, Y]) & =\kappa\left(\bar{\phi} h^{\prime}(\phi X, Y)-\bar{\phi} h^{\prime}(X, \phi Y)\right)+\kappa(X) \eta(Y)-\kappa(Y) \eta(X)
\end{aligned}
$$

Proof: Using (4.5) and (4.6), we obtain, for any $\mathrm{X}, \mathrm{Y} \in \Gamma\left(\mathrm{TM}^{\prime}\right)$,

$$
\begin{aligned}
& \mathrm{k}\left(\nabla_{\mathrm{X}}^{\prime} \mathrm{Y}\right)=\mathrm{g}\left(\mathrm{K}, \nabla_{\mathrm{X}}^{\prime} \mathrm{Y}\right)=\mathrm{v}(\mathrm{K}) \mathrm{u}\left(\bar{\nabla}_{\mathrm{X}} \mathrm{Y}\right)+\mathrm{u}(\mathrm{K}) v\left(\bar{\nabla}_{\mathrm{X}} \mathrm{Y}\right) \\
& =v(\mathrm{~K}) \mathrm{B}(\mathrm{X}, \phi \mathrm{Y})+\mathrm{u}(\mathrm{K}) \mathrm{C}(\mathrm{X}, \phi \mathrm{Y})+\mathrm{k}(\mathrm{X}) \eta(\mathrm{Y}) \\
& =-\kappa\left(\bar{\phi} h^{\prime}(X, \phi Y)\right)+\kappa(X) \eta(Y) \\
& \text { and } k([X, Y])=\kappa\left(\nabla_{X}^{\prime} Y\right)-\kappa\left(\nabla_{Y}^{\prime} X\right)=-\kappa\left(\bar{\phi} h^{\prime}(X, \phi Y)-\bar{\phi} h^{\prime}(Y, \phi X)\right)+\kappa(X) \eta(Y) \\
& -\kappa(Y) \eta(X)
\end{aligned}
$$

which completes the proof. 
Theorem 4.2. Let $(\mathrm{M}, \mathrm{g}, \mathrm{S}(\mathrm{TM}))$ be a lightlike hypersurface of an indefinite Kenmotsu manifold $(\overline{\mathrm{M}}, \overline{\mathrm{g}})$ with $\xi \in \mathrm{TM}$. Then, the distribution $\mathrm{D}_{\mathrm{O}} \perp\langle\xi\rangle$ is integrable if and only if

$$
\begin{aligned}
\mathrm{C}(\phi X, Y) & =\mathrm{C}(\mathrm{X}, \phi \mathrm{Y}), \quad \mathrm{B}(\phi \mathrm{X}, \mathrm{Y})=\mathrm{B}(\mathrm{X}, \phi \mathrm{Y}), \\
\text { and } \mathrm{C}(\mathrm{X}, \mathrm{Y}) & =\mathrm{C}(\mathrm{Y}, \mathrm{X}), \quad \forall \mathrm{X}, \mathrm{Y} \in \Gamma\left(\mathrm{D}_{0} \perp\langle\xi\rangle\right) .
\end{aligned}
$$

Proof: The proof follows from a direct calculation.

Note that when the distribution $D_{0}$ is integrable, the relations (4.20) and (4.21) are satisfied and vice versa.

Theorem 4.3. Let $(\mathrm{M}, \mathrm{g}, \mathrm{S}(\mathrm{TM}))$ be a lightlike hypersurface of an indefinite Kenmotsu manifold $(\overline{\mathrm{M}}, \overline{\mathrm{g}})$ with $\xi \in \mathrm{TM}$. Suppose the distribution $\mathrm{D}_{0} \perp\langle\xi\rangle$ is integrable. Let $\mathrm{M}^{\prime}$ be a leaf of $\mathrm{D}_{0} \perp\langle\xi\rangle$. Then

(i) If $\mathrm{M}^{\prime}$ is totally geodesic in $\mathrm{M}$, then $\mathrm{M}^{\prime}$ is auto-parallel with respect to the Levi-Civita connection $\nabla^{\prime}$ in $\mathrm{M}$ and $\bar{\phi}\left(\mathrm{TM}^{\perp}\right) \oplus \bar{\phi}(\mathrm{N}(\mathrm{TM}))$ is a Killing distribution on $\mathrm{M}^{\prime}$.

(ii) If $\mathrm{M}^{\prime}$ is parallel with respect to the Levi-Civita connection $\nabla^{\prime}$ in $\mathrm{M}$, then $\mathrm{M}^{\prime}$ is totally geodesic.

Proof: (i) Writing $Y \in \Gamma\left(D_{0} \perp\langle\xi\rangle\right)$ as $Y=\sum_{i=1}^{2 n-4} \frac{g\left(Y, F_{i}\right)}{g\left(F_{i}, F_{i}\right)} F_{i}+\eta(Y) \xi$, where $g\left(F_{i}, F_{i}\right) \neq 0$ and $\left\{F_{i}\right\}_{1 \leq i \leq 2 n-4}$ an orthogonal basis of $D_{0}$. So, it is easy to check that, for any $X, Y \in \Gamma\left(\right.$ TM $\left.^{\prime}\right)$, $h^{\prime}(X, \phi Y)=\sum_{i=1}^{2 n-4} \frac{g\left(Y, F_{i}\right)}{g\left(F_{i}, F_{i}\right)} h^{\prime}\left(X, \phi F_{i}\right)$. If $M^{\prime}$ is totally geodesic, then, for any $X, Y \in \Gamma\left(D_{0} \perp\langle\xi\rangle\right)$, $h^{\prime}(X, Y)=0$. In particular $h^{\prime}(X, \phi Y)=\sum_{i} \frac{g\left(Y, F_{i}\right)}{g\left(F_{i}, F_{i}\right)} h^{\prime}\left(X, \phi F_{i}\right)=0$. The auto-parallelism of $M^{\prime}$ follows from (4.18). Using (4.15), (4.18), (4.19) and the fact that $\mathrm{k}(\mathrm{X})=0, \forall \mathrm{X} \in \Gamma\left(\mathrm{D}_{0} \perp\langle\xi\rangle\right)$, we obtain $\left(\mathrm{L}_{K} \mathrm{~g}\right)(X, Y)=0$. So $\bar{\phi}\left(\mathrm{TM}^{\perp}\right) \oplus \bar{\phi}(\mathrm{N}(\mathrm{TM}))$ is a Killing distribution on $\mathrm{M}^{\prime}$. (ii) If $\mathrm{M}^{\prime}$ is parallel with respect to the connection in $M$, then, for any $X, Y, Z \in \Gamma\left(\mathrm{TM}^{\prime}\right),\left(\nabla_{X}^{\prime} \mathrm{h}^{\prime}\right)(\mathrm{Y}, \mathrm{Z})=0$. So, $\left(\nabla_{X}^{\prime} \mathrm{C}\right)(\mathrm{Y}, \mathrm{Z})-\mathrm{C}(\mathrm{Y}, \mathrm{Z}) \tau(\mathrm{X})=0$ and $\left(\nabla_{\mathrm{X}}^{\prime} \mathrm{B}\right)(\mathrm{Y}, \mathrm{Z})+\mathrm{B}(\mathrm{Y}, \mathrm{Z}) \tau(\mathrm{X})=0$. Using (3.7) and (3.19), since $\mathrm{D}_{0} \perp\langle\xi\rangle$ integrable, for $Z=\xi$, we have,

$$
0=\left(\nabla_{\xi}^{\prime} \mathrm{B}\right)(\mathrm{X}, \mathrm{Y})+\tau(\xi) \mathrm{B}(\mathrm{X}, \mathrm{Y})=-\mathrm{B}(\mathrm{X}, \mathrm{Y}) .
$$

Also, using (4.1), we obtain, for any $\mathrm{X}, \mathrm{Y} \in \Gamma\left(\mathrm{TM}^{\prime}\right)$,

$$
0=\left(\nabla_{\xi}^{\prime} C\right)(X, Y)-\tau(\xi) C(X, Y)=-2 C(X, Y)
$$

From (4.22) and (4.23), we get $h^{\prime}(X, Y)=0$ which completes the proof.

Note that, the Lie derivative (4.15) can be expressed in functions of Lie derivatives (3.12) and (3.29) as, for any $\mathrm{X}, \mathrm{Y} \in \Gamma(\mathrm{TM})$,

$$
\begin{aligned}
\left(\mathrm{L}_{\mathrm{K}} \mathrm{g}\right)(\mathrm{X}, \mathrm{Y})= & \mathrm{X}(\boldsymbol{v}(\mathrm{K})) \mathfrak{u}(\mathrm{Y})+\mathrm{Y}(v(\mathrm{~K})) \mathfrak{u}(\mathrm{X})+\mathrm{X}(\mathrm{u}(\mathrm{K})) v(\mathrm{Y})+\mathrm{Y}(\mathfrak{u}(\mathrm{K})) v(\mathrm{X}) \\
& +v(\mathrm{~K})\left(\mathrm{L}_{\boldsymbol{v}} \mathrm{g}\right)(\mathrm{X}, \mathrm{Y})+\mathfrak{u}(\mathrm{K})(\mathrm{Lug})(\mathrm{X}, \mathrm{Y}) .
\end{aligned}
$$


Theorem 4.4. Let $(\mathrm{M}, \mathrm{g}, \mathrm{S}(\mathrm{TM}))$ be a lightlike hypersurface of an indefinite Kenmotsu manifold $(\overline{\mathrm{M}}, \overline{\mathrm{g}})$ with $\xi \in \mathrm{TM}$. Suppose the distribution $\mathrm{D}_{0} \perp\langle\xi\rangle$ is integrable. Let $\mathrm{M}^{\prime}$ be a leaf of $\mathrm{D}_{0} \perp\langle\xi\rangle$. Then, the following assertions are equivalent:

(i) $\mathrm{M}^{\prime}$ is totally geodesic in $\mathrm{M}$,

(ii) $A_{\mathrm{E}}^{*} \mathrm{X}$ and $\mathrm{A}_{\mathrm{N}} \mathrm{X} \in \Gamma\left(\bar{\phi}\left(\mathrm{TM}^{\perp}\right) \oplus \bar{\phi}(\mathrm{N}(\mathrm{TM}))\right), \forall \mathrm{X} \in \Gamma\left(\mathrm{TM}^{\prime}\right)$,

(iii) $\bar{\phi}\left(\mathrm{TM}^{\perp}\right) \oplus \bar{\phi}(\mathrm{N}(\mathrm{TM}))$ is a Killing distribution on $\mathrm{M}^{\prime}$,

(iv) $\bar{\phi}\left(\mathrm{TM}^{\perp}\right)$ and $\bar{\phi}(\mathrm{N}(\mathrm{TM}))$ are Killing distribution on $\mathrm{M}^{\prime}$.

Proof: The equivalence of (i) and (ii) follows from direct calculations. Using the relation (4.24), we obtain the equivalence of (iii) and (iv). Next we prove the equivalence of (i) and (iii). Using the fact that $\kappa$ vanishes on $M^{\prime}$ and the relation (4.17), and since $D_{0} \perp\langle\xi\rangle$ is integrable, (4.15) becomes, for any $\mathrm{X}, \mathrm{Y} \in \Gamma\left(\mathrm{TM}^{\prime}\right)$,

$$
\begin{aligned}
\left(\mathrm{L}_{\mathrm{K}} \mathrm{g}\right)(\mathrm{X}, \mathrm{Y})= & -v(\mathrm{~K})\{\mathrm{B}(\mathrm{X}, \phi \mathrm{Y})+\mathrm{B}(\phi \mathrm{X}, \mathrm{Y})\}-\mathrm{u}(\mathrm{K})\{\mathrm{C}(\mathrm{X}, \phi \mathrm{Y}) \\
& +\mathrm{C}(\phi \mathrm{X}, \mathrm{Y})\}=-2 \mathrm{k}\left(\bar{\phi} \mathrm{h}^{\prime}(\mathrm{X}, \phi \mathrm{Y})\right)
\end{aligned}
$$

Suppose $M^{\prime}$ is totally geodesic in $M$. Then, $h^{\prime}(X, Y)=0$. In particular $h^{\prime}(X, \phi Y)=0$, since $\bar{\phi}\left(D_{0} \perp\langle\xi\rangle\right) \subset D_{0}$. Therefore $\left(L_{K} g\right)(X, Y)=0$, i.e. $\bar{\phi}\left(T M^{\perp}\right) \oplus \bar{\phi}(N(T M))$ is a Killing distribution on $\mathrm{M}^{\prime}$. The converse is obvious by (4.25).

Now, we deal with the geometry of the mean curvature vector of a leaf of a integrable distribution of a lightlike hypersurface $M$ of an indefinite Kenmotsu space form $\bar{M}(\mathrm{c})$. First of all, a submanifold $M$ is said to be totally umbilical lightlike hypersurface of a semi-Riemannian manifold $\bar{M}$ if the local second fundemental form $B$ of $M$ satisfies

$$
\mathrm{B}(\mathrm{X}, \mathrm{Y})=\rho g(X, Y), \quad \forall X, Y \in \Gamma(\mathrm{TM})
$$

where $\rho$ is a smooth function on $\mathcal{U} \subset M$. If $M$ is totally umbilical lightlike hypersurface of a semi-Riemannian manifold $\bar{M}$, then, we have $B(X, Y)=\rho g(X, Y)$, for any $X, Y \in \Gamma(T M)$, which implies, by using (3.7), that $0=B(\xi, \xi)=\rho$. Hence $M$ is totally geodesic.

It follows from this that a Kenmotsu $\bar{M}(\mathrm{c})$ does not admit any non-totally geodesic, totally umbilical lightlike hypersurface. From this point of view, Bejancu [1] considered the concept of totally contact umbilical semi-invariant submanifolds. The notion of totally contact umbilical submanifolds was first defined by Kon [10.

It is now important to investigate the parallelism of the nonzero mean curvature vector by regarding the effect of the totally contact umbilical condition on the geometry of lightlike submanifolds in Kenmotsu manifolds case. As it was done in case of lightlike hypersurfaces of indefinite Sasakian manifolds [15, the terminology of extrinsic sphere [3] will be used in case of totally contact geodesic submanifolds. We say that a totally contact umbilical submanifold is an extrinsic 
sphere when it has parallel non zero mean curvature vector [3]. In [15], the author showed that if $M$ is a totally contact umbilical lightlike hypersurface of $\bar{M}(\mathrm{c})$ with $\xi \in \mathrm{TM}$, that is, the second fundamental form $h$ of $M$ satisfies

$$
h(X, Y)=\{g(X, Y)-\eta(X) \eta(Y)\} H+\eta(X) h(Y, \xi)+\eta(Y) h(X, \xi),
$$

where $H=\lambda N$ normal vector field and $\lambda$ is a smooth function on $\mathcal{U} \subset M$, then $\lambda$ satisfies the partial differential equations 16

$$
\begin{array}{ll} 
& E(\lambda)+\lambda \tau(E)-\lambda^{2}=0, \quad \xi(\lambda)+\lambda(\tau(\xi)+1)=0, \\
\text { and } \quad & P X(\lambda)+\lambda \tau(P X)=0, \quad P X \neq \xi, \quad \forall X \in \Gamma(T M) .
\end{array}
$$

Some of these equations are similar to those of the generic submanifold of indefinite Sasakian manifolds case given in [13]. From the equations (4.28) and (4.29), the geometry of the mean curvature vector $H$ of $M$ is discussed. From (4.28) and (4.29), we have $\nabla_{\mathrm{E}}^{\perp} \mathrm{H}=\overline{\mathrm{g}}(\mathrm{H}, \mathrm{E})^{2} \mathrm{~N}$, $\nabla_{\bar{\xi}}^{\frac{1}{\xi}} \mathrm{H}=-\overline{\mathrm{g}}(\mathrm{H}, \mathrm{E}) \mathrm{N}$ and $\nabla_{\mathrm{PX}}^{\perp} \mathrm{H}=0, \mathrm{PX} \neq \xi, \forall \mathrm{X} \in \Gamma(\mathrm{TM})$. This means that $\mathrm{H}$ is not parallel on M.

Now, we prove that there exists a distribution which is a subset of the screen distribution $S(T M)$ in which, under the integrability condition, any totally contact umbilical leaf has a parallel nonzero mean curvature. Note that any totally contact umbilical leaf of an integrable screen distribution of a lightlike hypersurface of an indefinite Kenmotsu space form cannot be an extrinsic sphere [15]. Let us consider the following distribution

$$
\widehat{\mathrm{D}}=\left\{\bar{\phi}\left(\mathrm{TM}^{\perp}\right) \oplus \bar{\phi}(\mathrm{N}(\mathrm{TM}))\right\} \perp \mathrm{D}_{0}
$$

so that the tangent space of $M$ is written

$$
\mathrm{TM}=\widehat{\mathrm{D}} \perp\langle\xi\rangle \perp \mathrm{TM}^{\perp} .
$$

Now, referring to the decomposition (4.31), for any $X \in \Gamma(T M), Y \in \Gamma(\widehat{D})$, we have

$$
\nabla_{X} Y=\widehat{\nabla}_{X} Y+\widehat{h}(X, Y)
$$

where $\widehat{\nabla}$ is a linear connection on the bundle $\widehat{D}$ and $\widehat{h}: \Gamma(\mathrm{TM}) \times \Gamma(\widehat{\mathrm{D}}) \longrightarrow \Gamma\left(\langle\xi\rangle \perp \mathrm{TM}^{\perp}\right)$ is $\mathcal{F}(M)$-bilinear. Let $\mathcal{U} \subset M$ be a coordinate neighbourhood as fixed in Theorem 2.1. Then, using (4.31), (4.32) can be locally rewritten in the following way:

$$
\begin{aligned}
\nabla_{X} Y & =\hat{\nabla}_{X} Y+g\left(\nabla_{X} Y, \xi\right) \xi+g\left(\nabla_{X} Y, N\right) E \\
& =\widehat{\nabla}_{X} Y-g(X, Y) \xi+C(X, Y) E
\end{aligned}
$$

for any $X \in \Gamma(T M), Y \in \Gamma\left(\left.\widehat{D}\right|_{\mathcal{U}}\right)$ and the local expression of $\widehat{h}$ is

$$
\widehat{h}(X, Y)=-g(X, Y) \xi+C(X, Y) E .
$$


Lemma 4.4. Let $(\mathrm{M}, \mathrm{g}, \mathrm{S}(\mathrm{TM}))$ be a lightlike hypersurface of an indefinite Kenmotsu manifold $(\overline{\mathrm{M}}, \overline{\mathrm{g}})$ with $\xi \in \mathrm{TM}$. Then $\widehat{\mathrm{D}}$ is integrable if and only if

$$
\mathrm{C}(\mathrm{X}, \mathrm{Y})=\mathrm{C}(\mathrm{Y}, \mathrm{X}), \quad \forall \mathrm{X}, \mathrm{Y} \in \Gamma(\widehat{\mathrm{D}}) \text {. }
$$

Proof: The proof follows by direct calculation, using (4.33).

Theorem 4.5. Let $(M, g, S(T M))$ be a lightlike hypersurface of an indefinite Kenmotsu space form $(\overline{\mathrm{M}}(\mathrm{c}), \overline{\mathrm{g}})$ with $\xi \in \mathrm{TM}$ such that the distribution $\widehat{\mathrm{D}}$ is integrable. Suppose any leaf $\mathrm{M}^{\prime}$ of $\widehat{\mathrm{D}}$ is totally contact umbilical immersed in $\overline{\mathrm{M}}$ as non-degenerate submanifold. Then, $\mathrm{M}^{\prime}$ is an extrinsic sphere.

Proof: By combining the first equations of (2.7) and (2.8), we obtain

$$
\begin{aligned}
\bar{\nabla}_{\mathrm{X}} \mathrm{Y} & =\widehat{\nabla}_{\mathrm{X}} \mathrm{Y}-\mathrm{g}(\mathrm{X}, \mathrm{Y}) \xi+\mathrm{C}(\mathrm{X}, \mathrm{Y}) \mathrm{E}+\mathrm{B}(\mathrm{X}, \mathrm{Y}) \mathrm{N} \\
& =\widehat{\nabla}_{\mathrm{X}}^{\prime} \mathrm{Y}+\widehat{\mathrm{h}}^{\prime}(\mathrm{X}, \mathrm{Y}), \forall \mathrm{X}, \mathrm{Y} \in \Gamma\left(\mathrm{TM}^{\prime}\right)
\end{aligned}
$$

where $\widehat{\nabla}^{\prime}$ and $\widehat{h}^{\prime}$ are the Levi-Civita connection and second fundamental form of $M^{\prime}$ in $\bar{M}$.

Denote by $\mathrm{H}^{\prime}$ the mean curvature vector of $\mathrm{M}^{\prime}$. As $\mathrm{N}(\mathrm{TM}) \oplus \mathrm{TM}^{\perp}$ is the normal bundle of $M^{\prime}$, there exist smooth functions $\lambda$ and $\rho$ such that $H^{\prime}=\lambda E+\rho N$. Since $M^{\prime}$ is totally contact umbilical immersed in $\bar{M}$ we have

$$
\widehat{h}^{\prime}(X, Y)=(g(X, Y)-\eta(X) \eta(Y)) H^{\prime}+\eta(X) \widehat{h}^{\prime}(Y, \xi)+\eta(Y) \widehat{h}^{\prime}(X, \xi) .
$$

Since $\widehat{h}^{\prime}(X, \xi)=0$, for any $X \in \Gamma\left(\mathrm{TM}^{\prime}\right)$, from (4.35) we obtain

$$
\bar{\nabla}_{X} Y=\hat{\nabla}_{X}^{\prime} Y+(g(X, Y)-\eta(X) \eta(Y)) H^{\prime}
$$

which implies

$$
\begin{aligned}
\bar{\nabla}_{X} \bar{\nabla}_{Y} Z & =\widehat{\nabla}_{X}^{\prime} \widehat{\nabla}_{Y}^{\prime} Z+\left\{g\left(X, \widehat{\nabla}_{Y}^{\prime} Z\right)-\eta(X) \eta\left(\widehat{\nabla}_{Y}^{\prime} Z\right)\right\} H^{\prime} \\
& +\left\{g\left(\widehat{\nabla}_{X}^{\prime} Y, Z\right)+g\left(Y, \widehat{\nabla}_{X}^{\prime} Z\right)-\eta(Z) g(X, Y)+2 \eta(X) \eta(Y) \eta(Z)\right. \\
& \left.-\eta(Z) \eta\left(\widehat{\nabla}_{X}^{\prime} Y\right)-\eta(Y) g(X, Z)-\eta(Y) \eta\left(\widehat{\nabla}_{X}^{\prime} Z\right)\right\} H^{\prime} \\
& +\{g(Y, Z)-\eta(Y) \eta(Z)\} \bar{\nabla}_{X} H^{\prime} .
\end{aligned}
$$

Since $\widehat{D}$ is integrable, $\theta([X, Y])=0$, for any $X, Y \in \Gamma\left(\mathrm{TM}^{\prime}\right)$ and we have

$$
\bar{\nabla}_{[X, Y]} Z=\widehat{\nabla}_{[X, Y]}^{\prime} Z+\{g([X, Y], Z)-\eta([X, Y]) \eta(Z)\} H^{\prime} .
$$

From (4.38), (4.39) and (4.7)-(4.9), after calculations, we obtain

$$
\begin{aligned}
& \bar{R}(X, Y) Z=\widehat{R}^{\prime}(X, Y) Z+\{g(Y, Z) \eta(X)-g(X, Z) \eta(Y)\} H^{\prime} \\
& +\{g(Y, Z)-\eta(Y) \eta(Z)\} \bar{\nabla}_{X} H^{\prime}-\{g(X, Z)-\eta(X) \eta(Z)\} \bar{\nabla}_{Y} H^{\prime},
\end{aligned}
$$


where $\widehat{R}^{\prime}$ is the curvature tensor field of $M^{\prime}$.

Consequently,

$$
\begin{gathered}
\bar{g}(\bar{R}(X, Y) Z, E)=\{g(Y, Z) \eta(X)-g(X, Z) \eta(Y)\} \bar{g}\left(H^{\prime}, E\right)+\{g(Y, Z) \\
-\eta(Y) \eta(Z)\} \bar{g}\left(\bar{\nabla} X^{\prime} H^{\prime}, E\right)-\{g(X, Z)-\eta(X) \eta(Z)\} \bar{g}\left(\bar{\nabla}_{Y} H^{\prime}, E\right), \\
\bar{g}(\bar{R}(X, Y) Z, N)=\{g(Y, Z) \eta(X)-g(X, Z) \eta(Y)\} \bar{g}\left(H^{\prime}, N\right)+\{g(Y, Z) \\
-\eta(Y) \eta(Z)\} \bar{g}\left(\bar{\nabla}_{X} H^{\prime}, N\right)-\{g(X, Z)-\eta(X) \eta(Z)\} \bar{g}\left(\bar{\nabla}_{Y} H^{\prime}, N\right) .
\end{gathered}
$$

From (4.41) and using (2.3), we obtain

$$
\begin{aligned}
0= & \{g(Y, Z) \eta(X)-g(X, Z) \eta(Y)\} \bar{g}\left(H^{\prime}, E\right)+\{g(Y, Z) \\
& -\eta(Y) \eta(Z)\} \bar{g}\left(\bar{\nabla} \times H^{\prime}, E\right)-\{g(X, Z)-\eta(X) \eta(Z)\} \bar{g}\left(\bar{\nabla}_{Y} H^{\prime}, E\right) .
\end{aligned}
$$

Now, since $X, Y, Z \in \Gamma\left(\mathrm{TM}^{\prime}\right)$, the relation (4.43) is reduced as have

$$
\bar{g}\left(\bar{\nabla}_{X} H^{\prime}, E\right) Y=\bar{g}\left(\bar{\nabla}_{Y} H^{\prime}, E\right) X .
$$

Likewise, from (4.42) and (2.3), we have

$$
\bar{g}\left(\bar{\nabla}_{X} H^{\prime}, N\right) Y=\bar{g}\left(\bar{\nabla}_{Y} H^{\prime}, N\right) X .
$$

Now suppose that there exists a vector field $X_{0}$ on some neighborhood of $M^{\prime}$ such that $\bar{g}\left(\bar{\nabla}_{X_{0}} H^{\prime}, E\right) \neq 0$ and $\bar{g}\left(\bar{\nabla}_{X_{0}} H^{\prime}, N\right) \neq 0$ at some point $p$ in the neighborhood. From (4.44) and (4.45) it follows that all vectors of the fibre $\mathrm{TM}^{\prime}$ are collinear with $\left.\mathrm{X}_{0}\right|_{\mathrm{p}}$. This contradicts dim $\mathrm{TM}^{\prime}>$ 1. This implies $\overline{\mathrm{g}}\left(\bar{\nabla}_{\mathrm{X}} \mathrm{H}^{\prime}, \mathrm{E}\right)=0$ and $\overline{\mathrm{g}}\left(\bar{\nabla}_{\mathrm{X}} \mathrm{H}^{\prime}, \mathrm{N}\right)=0, \forall \mathrm{X} \in \Gamma\left(\mathrm{TM}^{\prime}\right)$. These lead, respectively, to $g\left(\widehat{\nabla}_{X}^{\prime \perp} H^{\prime}, E\right)=0$ and $g\left(\widehat{\nabla}_{X}^{\prime \perp} H^{\prime}, N\right)=0$, where $\hat{\nabla}^{\prime \perp}$ is a linear connection on $N(T M) \oplus T^{\perp}$ defined by $\widehat{\nabla}_{X}^{\prime \perp} \mathrm{E}=\nabla_{\mathrm{X}}^{* \perp} \mathrm{E}=-\tau(\mathrm{X}) \mathrm{E}$ and $\widehat{\nabla}_{\mathrm{X}}^{\prime \perp} \mathrm{N}=\nabla_{\mathrm{X}}^{\perp} \mathrm{N}=\tau(\mathrm{X}) \mathrm{N}$, which completes the proof.

We discuss here the effect of the change of the screen distribution (3.14) on all the results above. The Lie derivative (3.29) depends on $\mathrm{C}$ and $v$ which are not unique and their change can be seen as follows. Denote by $\omega$ the dual 1-form of $W=\sum_{i=1}^{2 n-1} c_{i} W_{i}$ with respect to the induced metric $g$ of $M$, that is $\omega(\cdot)=g(\cdot, W)$. Let $P$ and $P^{\prime}$ be projections of TM on $S(T M)$ and $S(T M)^{\prime}$, respectively with respect to the orthogonal decomposition of TM. So, any vector field $X$ on $M$ can be written as $X=P X+\theta(X) E=P^{\prime} X+\theta^{\prime}(X) E$, where $\theta^{\prime}(X)=\bar{g}\left(X, N^{\prime}\right)$. Then, using (3.14) we have $P^{\prime} X=P X-\omega(X) E$ and $C^{\prime}\left(X, P^{\prime} Y\right)=C^{\prime}(X, P Y), \forall X, Y \in \Gamma(T M)$. The relationship between the second fundamental forms $C$ and $C^{\prime}$ of the screen distribution $S(T M)$ and $S(T M)^{\prime}$, respectively, is given by

$$
C^{\prime}(X, P Y)=C(X, P Y)-\frac{1}{2} \omega\left(\nabla_{X} P Y+B(X, Y) W\right) .
$$

All equations above depending only on the local second fundamental form $C$ (making equations non unique) are independent of the screen distribution $S(T M)$ if and only if $\omega\left(\nabla_{X} P Y+B(X, Y) W\right)=$ $0, \quad \forall X, Y \in \Gamma(T M)$. 
The equations (3.29) and (4.15) also are not unique as they depend on C, $\theta$ and $\tau$ which depend on the choice of a screen vector bundle. The Lie derivatives $\mathrm{L}_{(\cdot)}$ and $\mathrm{L}_{(\cdot)}^{\prime}$ of the screen distributions $S(T M)$ and $S(T M)^{\prime}$, respectively, are related through the relations:

$$
\begin{aligned}
& \left.\left(\mathrm{L}_{\mathrm{u}^{\prime}}^{\prime} \mathrm{g}\right)(\mathrm{X}, \mathrm{Y})=\left(\mathrm{Lug}_{\mathrm{ug}}\right)(\mathrm{X}, \mathrm{Y})+\frac{1}{2} \omega\left(\nabla_{\{\mathrm{X}} \mathrm{P} \phi \mathrm{Y}\right\}+(\mathrm{B}(\mathrm{X}, \phi \mathrm{Y})+\mathrm{B}(\phi X, \mathrm{Y})) \mathrm{W}\right) \\
& +\frac{1}{2}\{\eta(X) \omega(-2 \phi Y+u(Y) W)+\eta(Y) \omega(-2 \phi X+u(X) W)\} \\
& -\frac{1}{2}\{\tau(X) \omega(-2 \phi Y+u(Y) W)+\tau(Y) \omega(-2 \phi X+u(X) W)\}+v_{X}(Y) \\
& -\frac{1}{2}\{B(X, W) \omega(-2 \phi Y+u(Y) W)+B(Y, W) \omega(-2 \phi X+u(X) W)\}, \\
& \left(\mathrm{L}_{\mathrm{K}^{\prime}}^{\prime} \mathrm{g}\right)(\mathrm{X}, \mathrm{Y})=\left(\mathrm{L}_{\mathrm{K}} \mathrm{g}\right)(\mathrm{X}, \mathrm{Y})+\left(\boldsymbol{v}^{\prime}\left(\mathrm{K}^{\prime}\right)-v(\mathrm{~K})\right)\left(\left(\mathrm{L}_{\boldsymbol{v}} \mathrm{g}\right)(\mathrm{X}, \mathrm{Y})+\mathrm{u}\left(\mathrm{X}_{(\tau+\eta)(\mathrm{Y})}\right)\right) \\
& +\left(u\left(K^{\prime}\right)-u(K)\right)\left(\left(L_{u g}\right)(X, Y)+v\left(X_{(\eta-\tau)(Y)}\right)\right)+\eta\left(X_{\left(\kappa-K^{\prime}\right)(Y)}\right) \\
& \left.+\frac{1}{2} \mathfrak{u}\left(\mathrm{K}^{\prime}\right) \omega\left(\nabla_{\{X} \mathrm{P} \phi Y\right\}+(\mathrm{B}(\mathrm{X}, \phi Y)+\mathrm{B}(\phi X, Y)) W\right)
\end{aligned}
$$

where $\left.f_{X}(Y)=f(X) B(Y, W)+f(Y) B(X, W), \nabla_{\{X} P Y\right\}=\nabla_{X} P Y+\nabla_{Y} P X, v^{\prime}(X)=v(X)-\frac{1}{2} \kappa(-2 \phi X+$ $u(X) W)$ and $X_{f(Y)}=X f(Y)+Y f(X), f$ denoting a 1-form.

Received: August 2009. Revised: August 2010.

\section{References}

[1] A. Bejancu, Umbilical Semi-invariant submanifolds of a Sasakian manifold, Tensor N. S., 37 (1982), 203-213.

[2] D. E. Blair, Riemannian Geometry of Contact and Symplectic Manifolds, Progress in Mathematics 203. Birkhauser Boston, Inc., Boston, MA, 2002.

[3] M. Dajczer et al., Submanifolds and isometric immersions, Mathematics lecture series 13. Publish or Perish, Inc., Houston, Texas, 1990.

[4] K. L. Duggal and A. Bejancu, Lightlike submanifolds of semi-Riemannian manifolds and applications, Mathematics and Its Applications. Kluwer Publishers, 1996.

[5] K. L. Duggal and D. H. Jin, Null curves and hypersurfaces of semi-Riemannian manifolds, World Scientific Publishing Co. Pte. Ltd. 2007.

[6] K. L. Duggal and B. Sahin, Lightlike Submanifolds of Indefinite Sasakian Manifolds, Internat. J. Math. Math. Sci., vol. 2007, Article ID 57585, 21 pages.

[7] J-B. Jun, U. C. De and G. Pathak, On Kenmotsu manifolds, J. Korean Math. Soc., 42 (3) (2005), 435-445.

[8] D. Janssens and L. Vanhecke, Almost contact structures and curvature tensors, Kodai Math. J., 4 (1981), 1-27. 
[9] K. Kenmotsu, A class of almost contact Riemannian manifolds, Tohoku Math. J., 24 (1972), 93-103.

[10] M. Kon, Remarks on anti-invariant submanifold of a Sasakian manifold, Tensor, N. S., 30 (1976), 239-246.

[11] F. Massamba, A note on Umbilical lightlike hypersurfaces of indefinite Sasakian manifolds, Int. J. Contemp. Math. Sciences, 2, 32 (2007), 1557-1568.

[12] F. Massamba, Lightlike hypersurfaces of indefinite Sasakian manifolds with parallel symmetric bilinear forms, Differ. Geom. Dyn. Syst., 10 (2008), 226-234.

[13] F. Massamba, Totally contact umbilical lightlike hypersurfaces of indefinite Sasakian manifolds, Kodai Math. J., 31 (2008), 338-358.

[14] F. Massamba, Screen integrable lightlike hypersurfaces of indefinite Sasakian manifolds, Mediterr. J. Math., 6 (2009), 27-46.

[15] F. Massamba, On Lightlike geometry in indefinite Kenmotsu manifolds, to appear in Mathematica Slovaca.

[16] F. Massamba, On semi-parallel lightlike hypersurfaces of indefinite Kenmotsu manifolds, $J$. Geom, 95 (2009), 73-89. 